Florida International University FIU Digital Commons

FIU Electronic Theses and Dissertations

University Graduate School

6-17-1988

\title{
An expert system for project controls in construction management
}

Kermit H. Gates

Florida International University

DOI: $10.25148 /$ etd.FI15101544

Follow this and additional works at: https://digitalcommons.fiu.edu/etd

\section{Recommended Citation}

Gates, Kermit H., "An expert system for project controls in construction management" (1988). FIU Electronic Theses and Dissertations. 3588.

https://digitalcommons.fiu.edu/etd/3588

This work is brought to you for free and open access by the University Graduate School at FIU Digital Commons. It has been accepted for inclusion in FIU Electronic Theses and Dissertations by an authorized administrator of FIU Digital Commons. For more information, please contact dcc@fiu.edu. 


\begin{abstract}
AN EXPERT SYSTEM FOR PROJECT CONTROLS

IN CONSTRUCTION MANAGEMENT
\end{abstract}

by

Kermit H. Gates

In this paper, I describe an expert project control system for construction management. The purpose of the project is to develop methods and strategies for expert system based planning, scheduling, chronicling and analysis for construction management. Planning defines the actions required to accomplish a goal; scheduling links the plan into a frame of time; chronicling is monitoring job performance and analysis defines reevaluation of the plan as conditions change. Conditions are modeled as constraints and will be coded as rules. As conditions change, constraints must be dynamically modified by the system to accommodate the changes. The research is a combination of three related areas:

a. Domain dependent hierarchical planning techniques.

b. Model-based planning/scheduling techniques developed for the job-shop environment.

c. Expert construction planning/scheduling techniques. 
AN EXPERT SYSTEM FOR PROJECT CONTROLS

IN CONSTRUCTION MANAGEMENT

by

Kermit H. Gates

A thesis submitted in partial fulfillment of the requirements for the degree of MASTER OF SCIENCE

in

COMPUTER SCIENCE

at

FLORIDA INTERNATIONAL UNIVERSITY

Committee in charge:

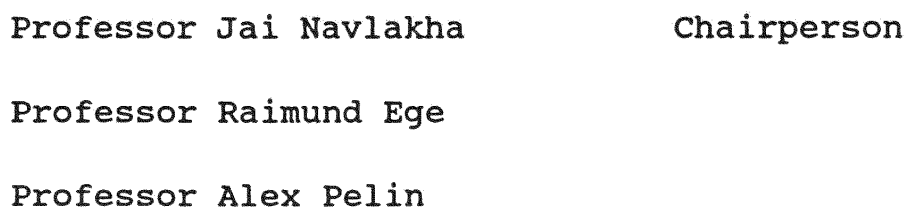

June 1988 
To Professors Jai Navlakha, Raimund Ege and Alex Pelin

This thesis, having been approved in respect to form and mechanical execution, is referred to you for judgment upon its substantial merit.

\author{
Dean James A. Mau \\ College of Arts and Sciences
}

The thesis of Kermit H. Gates is approved.

Professor Raimund Ege

Professor Alex Pelin

Major Professor Jai Navlakha

Date of Examination:

June 17,1988 


\title{
AN EXPERT SYSTEM FOR PROJECT CONTROLS
}

\section{IN CONSTRUCTION MANAGEMENT}

$$
\text { by }
$$

\author{
Kermit H. Gates
}

\begin{abstract}
A thesis submitted in partial fulfillment of the requirements for the degree of

MASTER OF SCIENCE

in

COMPUTER SCIENCE
\end{abstract}

at

FLORIDA INTERNATIONAL UNIVERSITY

1988 


\section{ACKNOWLEDGEMENTS}

I would like to thank Dr. Jai Navlakha and Dr. Mihaly Lenart for their support in this endeavor. I am also blessed with a patient and loving wife, without whose continued encouragement this goal would never have been accomplished. 
TABLE OF CONTENTS

LIST OF FIGURES $\ldots \ldots \ldots \ldots \ldots \ldots \ldots \ldots \ldots \ldots \ldots$ vi

Chapter Page

1. INTRODUCTION $\ldots \ldots \ldots \ldots \ldots \ldots \ldots \ldots \ldots \ldots \ldots$

Knowledge Representation $\ldots \ldots \ldots \ldots \ldots \ldots \ldots$

Semantic Nets

Structured objects - Frames

AI Planning Models ................ 10

Means-end Analysis

NOAH and Hierarchical Planning

NONLIN

Blackboard Architecture

Knowledge Processing Systems

The Construction Management Problem....... 23

ART Expert System Shell............. 26

2. SYSTEM FEATURES $\ldots \ldots \ldots \ldots \ldots \ldots \ldots \ldots \ldots \ldots . \ldots . \ldots . \ldots$

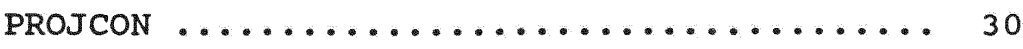

Strategic Planning

Project Planning 
Project scheduling

Project Chronicling and Analysis

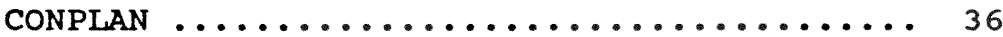

System structure

Model Hierarchy

Phased Implementation of Conplan

Phase I - Object Model, User Interface

Phase II - Action Model

Phase III - Partial Ordering

Phase IV - Evaluate Parallel Actions

3. SYSTEM DESIGN - CONPLAN $\ldots \ldots \ldots \ldots \ldots \ldots \ldots \ldots \ldots 2$

Rule-Based Programming ............. 52

CONPLAN system specifications $\ldots \ldots \ldots \ldots \ldots 55$

Functional Specifications

Phase I External Specifications

Phase II External Specifications

Phase III External Specifications

Phase IV External Specifications

General Model Internal Specifications

Phase I Internal Specifications 
Phase II Internal Specifications

Phase III Internal specifications

Phase IV Internal specifications

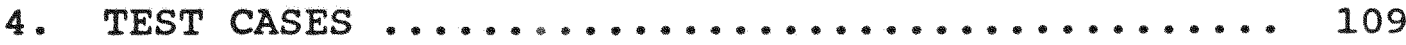

Program Attributes to be Tested .......... 109

Test Cases Chosen ....................... 112

TEST-CASE1

TEST-CASE2

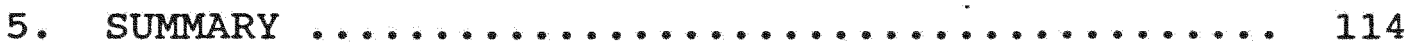

REFERENCE LIST $\ldots \ldots \ldots \ldots \ldots \ldots \ldots \ldots \ldots \ldots \ldots \ldots \ldots \ldots \ldots \ldots \ldots$ 
LIST OF FIGURES

Figure

Page

1. Model Hierarchy $\ldots \ldots \ldots \ldots \ldots \ldots \ldots \ldots \ldots . \ldots . \ldots$

2. Organization Model Schema ............ 40

3. Environment Model Schema ............. 41

4. Physical Object Model Schema ........... 42

5. Action Model Schema $\ldots \ldots \ldots \ldots \ldots \ldots \ldots \ldots$

6. State Model schemata ............... 44

7. Sample Ternary Fact $\ldots \ldots \ldots \ldots \ldots \ldots \ldots \ldots \ldots$

8. Sample ART Rule ............... 55

9. CONPLAN Initial screen $\ldots \ldots \ldots \ldots \ldots \ldots \ldots 60$

10. Screen Layout For Structural Wall Selection 62

11. Screen Used For Room Function Selection .... 66

12. Phase II Initial Screen $\ldots \ldots \ldots \ldots \ldots \ldots \ldots 68$

13. Resource Availability Constraint Input .... 71

14. General object Model Hierarchy ......... 75

15. One Category From object Model ......... 77

16. Action Model Hierarchy $\ldots \ldots \ldots \ldots \ldots \ldots . \ldots 79$

17. Sample Actions At Model Leaves .......... 80

18. Sample schema $\ldots \ldots \ldots \ldots \ldots \ldots \ldots \ldots \ldots \ldots$ 
19. Semantic Net Representation of Schema ..... 81

20. Sample Relation Definition ........... 82

21. Inheritance Properties ............ 85

22. Object Model Instance TEST-CASE1 ....... 88

23. Explicit And Inherited Model Ties ....... 89

24. Object Model Instance TEST-CASE2 ........ 91

25. Walls Hierarchy For TEST-CASE1 ........ 92

26. Relational Tie Between Action Models ...... 98

27. Building Prototype Structural Properties ... 103 
CHAPTER 1

\section{INTRODUCTION}

PROJCON, (Project control) uses frame-based hierarchical knowledge representation methods recently applied to manufacturing by Sathi [33], hierarchical planning techniques originally developed by Sacerdoti [31] and constraint-directed search techniques used by Fox [10]. A fundamental difference between PROJCON and previous work is PROJCON's automated planning mechanism. PROJCON allows a user to graphically depict a building using geometric objects and then develops a project plan using methods from a hierarchical frame-based action model. These methods show how individual sections of a building would be constructed if no outside influences were present. PROJCON then evaluates the actions in the context of the particular building to determine interferences and search the resulting graph for an appropriate solution. The system also allows a user to specify global constraints that are used in prioritizing actions in the plan. For example, if a user specifies that one section of a building should be completed ahead 
of others, that requirement will be coded as a rule and the required actions are given higher priority.

CONPLAN (Construction Planning) is the portion of PROJCON that includes a graphical user interface, a plan generation system and a resource evaluation system. The graphical user interface allows a database to be generated that describes a single story commercial building constructed of concrete, concrete block or wood frame as specified by the South Florida Building Code [37]. From this database, methods are chosen that show how each part of the building could be constructed, if they were built independently of one another. ordering rules are then applied that show the order of implementation of each part relative to other building parts. Finally, another rule base evaluates the network based upon available resources. The resulting graph can be input to a commercially available critical path method (CPM) scheduling system as used on most construction projects.

\section{Knowledge Representation}

Knowledge representation deals with structuring information so that domain dependent problems can be 
solved within the context of specific problem solving methodologies. There are three requirements for knowledge representation: a representation language, an inference method and domain information. In the case of knowledge representation, I found no papers detailing different representation techniques relating to construction management. As such, the following sections are primarily related to the computer science aspects of knowledge representation. I have included this section because of its importance to my thesis topic.

A knowledge representation language must, in a flexible and modular way, express the facts needed to make a reasonable decision in a complex environment. The language must be flexible enough to allow the complete semantics of a problem domain to be expressed. The language must be modular to allow program design simplicity as well as a context for variable binding. A context for variable binding defines the extent to which a local variable has meaning. 


\section{Semantic Nets}

Semantic nets were first initiated by Quillian [29] in an application intended to describe semantic representation of natural language. In his Ph.D. thesis, Quillian used nodes in a network to represent word concepts and links to highlight other related concepts. Each node (word) was head of a "plan" of nodes, whose structure contained the definition for the word. A definition for a word was determined by traversing links emanating from the word node to other word nodes. As such, the definition of one word was comprised of one or many other words (as you would expect). Quillian also wanted his representation to serve as a general inferential model for knowledge. He described an intersection search, which could show possible relations between words by performing a breadth-first traversal of the network surrounding the words.

Carbonell [5] expanded on Quillian's ideas by introducing concept units and example units. This was the first use of instantiation (although it would not be called instantiation for some time). Concept units contained abstract information that was common to a class 
of objects. Example units, on the other hand, were specific instances of objects that were members of the set described by concept units. Example units had all the characteristics of concept units and could add specific information not required of the more general concept units.

Shapiro [35] was first to generalize the definition of nodes and relations in a semantic net. Previous to this work, there was no concept of a standard semantic net definition. Hendrix [17] introduced the concept of partitioning to semantic nets. Partitions are a classification of concepts into related groups. This allowed for universally quantified objects to be shared by a group of concepts. It also formalized a way to differentiate between universally and existentally quantified objects by utilizing a "form" relation to isolate the partition in which universally quantified objects belong.

Brachman [4] extended semantic net concepts by defining epistemological properties of his layered representation. The structure he presented defined a "predetermined internal organization that is more 
sophisticated than sets of cases", refering to a case structure introduced in earlier work. He also described a layered approach to semantic nets, having five distinct levels. The implementational layer was made up of the data structures required to implement the network. The logical layer provides logical operators, propositions and predicates. It provides the logical mechanisms that are used in the next higher layer to define inference techniques. The epistemological layer contains concept types, inheritance mechanisms and classifies structural relations. The conceptual layer provides primitive relations, actions and object definitions. The linguistic layer defines the words, concepts and expressions needed for a particular knowledge domain.

Each layer in the hierarchy is built using capabilities defined at successively lower levels and individual layers are defined without knowledge of higher levels. For instance, the logical layer does not make use of the concepts defined in the (higher) epistemological layer. The epistemological layer should only apply concepts defined at lower levels in its own definition. 


\section{Structured objects - Frames}

According to Minsky [24], a frame is "a data structure for representing a stereotyped situation". Information stored within a frame describes an object, its current state and possible state transitions. Frame systems are hierarchies of frames that are grouped according to a classification for a specific problem domain. In such systems a frame can represent an object, a group of objects or an abstract concept that describes behavior for an object.

In the planning problem, frame systems can represent a search space from which a plan can be found (by constraint directed search for instance). Minsky made the analogy beween frame systems and human thinking by suggesting that human problem solving techniques could be based upon the concept of frames.

A frame data structure is made up of slots (relations) that point to other frames or procedures. In the simplest form of a slot, a value can be stored that describes a characteristic of the object. Each slot (or relation) is also a frame whose characteristics show the number of values a slot can contain and its inheritance 
properities. If a slot is an inheritance relation, the information at one node may be also known at other nodes. How much information is shared with other nodes is determined by the definitions of other slots in the node. slots can be defined so that information stored will not be shared with other nodes, even if an inheritance slot is present. In Pascal terms, a frame can be considered as a record of elements. Each record has a name and each element is a slot characteristic. A major difference is that dynamic instantiation of record variables in a pascal program is not possible, while dynamic generation of structured objects is a desired property of a knowledge representation language. Additionally, Pascal record fields have no predefined meaning, whereas in a semantic net a slot can carry a meaning, such as "inheritance slot".

Relational slots are generally of two types: structural or inheritance. Structural relations are ties between related objects and are used in the search process to traverse a network. Structural relations or usually horizontal in nature, tying together information at similar levels of abstraction. Inheritance relations, on 
the other hand, are generally vertical ties between objects of differing abstraction levels. They allow information stored in objects of higher abstraction to be "known" at lower (more concrete) levels in the hierarchy. For instance, if an object is-a red ball, then all the characteristics of "ball" would be inherited by the red bal1. This is also an example of instantiation where a red ball is a member of the "ball" set.

Information stored in a slot can name a procedure so that a range of values can be represented. A "deamon" (or condition) triggers the procedure to activate, allowing some action to change the value stored in the slot. A "deamon" is a mechanism that wakes the procedure when the proper circumstances are present. For instance, a rule could contain a pattern that will be matched when a ball is kicked (a state), activating a graphic procedure that will change the location of the red ball on a terminal screen.

The simplest form of slot information is a value. In this case an attribute for an object is specified. To describe a ball, for example, one slot called "has-color" 
could contain the value "red", while another, "diameter" could contain the value 16 (its diameter in inches).

\section{AI Planning Models}

The following sections describe various AI planning methodologies and systems. There are three general categories for planning systems: general purpose planners using little domain dependent knowledge; planners utilizing a combination of domain knowledge and general purpose planning strategies; and those that are mostly domain dependent problem solving tools. General purpose planners are typified by those that use means-end analysis as their search strategy. NOAH (Sacerdoti [31]) was one system in which means-end analysis was combined with hierarchial structures and least commitment decisionmaking principles. Planners using a combination of general purpose planning techniques coupled with domain dependent knowledge can be typified by the blackboard approach to problem solving. The blackboard approach to problem solving is made up of three major components: knowledge sources, a global data store and a control structure. The control structure allows problem solving 
methods stored in the knowledge sources to incrementally change the state of the global data store in an opportunistic manner, until a goal state is achieved. The OPM system (Hayes-Roth [15]) will be used as an example of the blackboard architecture.

\section{Means-end Analysis}

Means-end analysis is a problem solving methodology based upon problem-reduction. In problem reduction, a large problem can be viewed as a conjunction of several subproblems that can be solved independently. A large problem can be viewed as a graph of nodes, each representing a subproblem, in a hierarchical fashion. solutions to each subproblem are described using additional levels of nodes, each level being the solution to the subproblem defined by its parent. The search space can be represented as an and/or graph, with conjunctions ("and" links) being the subproblems needed to solve a particular problem and disjuctions ("or" links) representing alternative approaches to solving a subproblem. An and/or graph node is considered to contain a solution if: 
a. It is a terminal node (and thus has a solution)

b. It is an "or" node and at least one of the children is solved.

c. It is an "and" node and all of its children are solved.

Means-end analysis is applicable to problems where the solution is represented as a graph, as opposed to a single object or a path through a graph. In the planning problem, just such a solution representation can be used. The plan can be a directed, acyclic graph with actions as nodes and links showing the order in which the actions must be accomplished. An and/or graph can be searched to determine if a solution exists for a given situation. The plan would therefore be a subgraph (or subgraphs) within the and/or graph solution space.

\section{NOAH and Hierarchical Planning}

NOAH is a computerized planning system developed by Sacerdoti [31] in 1975. It is a system that elaborates goals into subgoals in a recursive manner, continually refining the level of detail in a plan until an acceptable plan is developed (no more rules can fire). A procedural 
net is developed that represents the hierarchy of detail levels and models procedural as well as declarative knowledge. Procedural knowledge is the capability to call functions that expand a goal into subgoals. Procedural knowledge also models the actions of operators on the system state. Declarative knowledge is the inferences achieved as a result of the procedural expansion of the previous goal into subgoals.

The solution to a problem begins as a single goal node. Functions are called that can break the single (abstract) goal into smaller more easily solved subgoals. At the same time, these newly created subgoals are linked to their parent node and become themselves goals in the next level of expansion. At some point, the level of detail required to express the problem is achieved and this process halts. The final network of nodes is the procedural net and represents a manner in which the initial problem can be solved.

Subgoals at each level are evaluated to ensure that conflicting subgoals do not exist. "Critic programs" are used to evaluate subgoals in this manner. When a new level of subgoals has been generated, critic programs 
evaluate the newly elaborated subgoals for inconsistencies and interaction problems before beginning the next level of expansion. Backtracking is not eliminated by this method, but the amount of backtracking is minimized.

Critic programs can be categorized as being in one of two classes: general purpose and task specific. The following are some general purpose critic programs used by NOAH. The "resolve conflicts" critic evaluates a plan and determines areas of conflict between parallel paths within a plan. For instance, if two subplans occur concurrently, and both required the same resources (tool, material or labor resources) then a conflict is found, and the subplans are replanned in series.

The "use existing objects" critic trys to bind previously unbound variables to objects already identified in the network. As the planning procedes in NOAH, objects will not be bound to subgoals unless a specific reason exists to do so. If not bound, a formal object (unbound object) is used instead. At a later point in time, the "use existing objects" critic will attempt to bind formal objects with objects already defined within the subnet. As an example, if one subplan requires a ladder (object) 
in a specific location and another parallel subplan (assuming no conflict) calls for the use of the same object, but does not specify its location, the latter subplan will have its formal object (location in this case) bound to that of the first subplan.

The "eliminate redundant preconditions" critic checks that no parallel subplans have the same preconditions. If two subplans have the same requirement in order to begin their execution, one precondition is removed and both subplans are linked to the remaining identical precondition.

The "resolve double cross" critic deals with conflicts in which two conjunctive subgoals prevent preconditions required for each other. One subgoal needs a condition to be true, while the other requires it to be false, preventing either from occuring (catch 22). The solution involves determining the source of conflict and modifying the subplans in order to prevent the conflict from arising.

General purpose critics are designed to be generic critic programs, useful in many circumstances. Task specific critics, on the other hand, are user defined 
network analysis programs that consider knowledge that is specific to a domain, allowing a user to tailor the system to specific requirements.

NOAH's drawback was that it could not guarrantee that a solution could be found, even if one existed. Although it applied the concept of delayed binding to its decision making process, once the decision was accepted it could not be reversed. In this manner, an incorrect search path could be traversed resulting in no solution being found.

\section{NONLIN}

NONLIN was developed by Austin Tate [41] at the University of Edinburgh. It followed the NOAH framework, investigating the use of partially ordered networks in a planning system combining $A I$ and operations research techniques. NONLIN followed NOAH's hierachical planning methodology of delayed binding for precedence decisions. It enhanced the formalism by allowing a decision to be reversed if conditions warranted. NOAH delayed the decision-making process, but once a decision was made, it was not reversible. As such, it was possible for NOAH to realize late in the process that an error had occured 
which could not be changed. NONLIN, on the other hand, allowed two possibilities at any decision point. It could reverse a precedence decision, backtrack to the point of indecision and continue down a different solution tree path. In this manner, the incompleteness of NOAH could be avoided.

\section{Blackboard Architecture}

Blackboard planning systems utilize a combination of domain knowledge (knowledge bases) and general purpose planning strategies (a control structure). The blackboard methodology for problem solving is a special structured case of opportunistic problem solving. Several knowledge sources contain information that is specific to one aspect of solving a large problem. This information classification allows a control structure to call into play the most efficient methods to solve a subproblem related to the current system state. The blackboard model is generally comprised of three major parts (Nii [26]): knowledge sources, the blackboard data structure and a control method. The knowledge sources are structured around a domian dependent problem solving methodology that 
are kept separate and independent. Information stored there is used to incrementally solve a problem. Knowledge sources are represented as procedures, rules or logical assertions.

The blackboard data structure is the common link between knowledge sources and the control method. The blackboard contains the current system state and changes incrementally from initial state to final goal (or goals). It holds hierarchically organized levels of objects that form the solution space. Information at one level can be used as input to knowledge sources which can in turn place their output at the same or different levels of the blackboard. Thus, the blackboard acts as an indirect communication device for knowledge sources. Solution spaces can be depicted as a hierarchy of abstractions. Each successively higher solution space in this structure contains progressively more abstract information.

The control structure allows the application of knowledge sources in an opportunistic manner. It monitors the blackboard state, deciding which knowledge base would best solve the current subproblem depicted there. The control structure also decides which solution space to 
pursue based upon dynamic blackboard changes caused by knowledge sources.

\section{Knowledge Processing Systems}

Knowledge processing systems utilize domain dependent information in three ways: by interpreting performance data to make probabilistic decisions; by constraining the search process and thereby limiting the search space; by using hierarchical model-based systems to allow a system to infer knowledge about underlying processes during decision making.

As mentioned earlier in this paper, hierarchical planning was elaborated by Sacerdoti [31] at stanford University. His methodology used domain-specific knowledge to expand (recursively) an abstract goal into subgoals or actions into component actions. At successive levels in the hierarchy, goals are expanded into subgoals until the problem has been completely described in terms of atomic actions. Evaluation functions are used to determine if competing actions cause conflicts in a plan.

Fox [10] and smith [38] expanded and applied hierarchical knowledge representation and search 
techniques to job-shop scheduling. Sathi [31] further applied those techniques to project management for the job-shop environment. Job-shop scheduling is a specific class of the production planning and scheduling problem. Sathi used a frame data structure (first described by Minsky [24]) to elaborate characteristics about objects at differing levels of abstraction. Using knowledge representation techniques, general information defined by prototypes can be inherited at lower (less abstract) levels in the hierarchy.

Sathi references a five layered semantic net model for project management that was originally defined by Brachman [4] as a general semantic net model (described in an earlier section in this paper) and by Fox [11] who adapted the model to the job shop environment:

a. Domain layer: concepts and knowledge relating to a specific project.

b. Semantic layer: defines concepts common to any project.

c. Epistemological layer: distinguishes the classes of frames and their slots. 
d. Logical layer: allows an interpretation of the information in each slot through logical inference..

e. Implementation layer: defines the frame and its slots as the lowest level data structures in the knowledge base.

An application dealing specifically with the construction management problem was developed by Levitt [20] at Stanford University. The system, called PLATFORM, whose domain is off-shore oil platforms, analyzes historical information to help determine "knights" and "villains" that could affect a construction schedule. A knight is a situation that could change a plan in an optimistic way, while a villain could raise doubt as to the plan's feasability. By quantifying the results of these forces using probabilistic methods, a schedule can be modified based upon prevailing jobsite conditions. Levitt theorizes that this is how a construction manager uses trained eyes to place emphasis on situations with the greatest potential impact on the project.

PLATFORM is written in KEE, a hybrid software environment integrating frame-based representation, rule based reasoning, active images and active values with LISP 
as the underlying programming language. It also uses interactive graphics for representing and modifying project schedules. The latest member of the PLATFORM family, PLATFORM III uses "multiple worlds" helping project managers in decision making that involve uncertainities. For this purpose it makes use of Dekleer's Assumption Based Truth Maintanance system (DeKleer [9]) by allowing the user to make assumptions about hypothetical decisions and situations.

Although PLATFORM (or rather the family of PLATFORMs) seems to be the only closely related project to our domain, there are several expert systems dealing with problems in construction management (see Kim [18]). Some of them are solving problems related to project control such as MASON (Hendrickson [16]), an expert system for estimating masonry construction durations; Gray [13], an expert system developed to help designers evaluate different construction methods; o'Connor [28], an expert system that analyzes construction schedules in order to maintain contracts, payments and job dead-1ines. 


\section{The Construction Management Problem}

Project control for construction management is

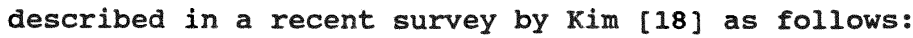

a. Developing time and estimates of construction tasks, particularly in the early stages of project planning;

b. Allocating constrained resources to activities;

c. Monitoring time and resource consumption;

d. Diagnosing reasons for cost, time or resource overruns, forecasting, durations and costs of remaining activities on projects; and

e. Developing remedial actions.

Construction management combines classical network scheduling methods with construction practices and techniques. Classical network scheduling methods include CPM and PERT (see Malcolm [23] and Levy [22]). Critical path method (CPM) takes as input a directed acyclic graph containing actions and a precedence relation and determines the longest path through the network (termed the critical path). CPM assigns a float value to paths that are not critical, giving the manager of a large project a way to prioritize actions and maximize organizational effectiveness. Program evaluation and 
review technique (PERT) differs from CPM in that it considers three different durations for each activity: optimistic, pessimistic and most likely.

Construction management (see Barrie [3]) is a domaindependent application of general project management. In construction management, an individual or a team of professionals is responsible to coordinate designers, contractors, material suppliers and financiers during construction of a project. Sathi [32] gives a convenient outline for project control in terms of its functions and management levels. The functional requirements are:

a. Objective setting - initial decisions based upon the specific job to be constructed. Also includes an organization's strategic planning as well as tactical planning requirements.

b. Project Planning - generating a network of actions.

c. Project scheduling - linking a plan into a frame of time as well as smoothing out resource peaks.

d. Chronicling and Analysis - measuring job progress and replanning, rescheduling when conditions change. 
PROJCON applies expert problem-solving techniques to each functional aspect of project management, as will be borne out in the next section.

In addition to functional aspects, construction management is tied to information flow between executives, responsible for strategic planning, and midlevel managers, responsible for tactical implementation of strategic plans. Similarly, the plan and schedule should also be structured in a hierarchical fashion to facilitate and simulate information flow at a construction project. Typically, management levels are defined by (see Levitt [20]):

a. Executive level - summary level reports showing major activities only.

b. Work Package level - activities at the executive level have little detail. At this level, they are broken into more detailed actions for a midlevel manager to control.

c. Task level - used at the craftsman level to show exactly what must be accomplished on a day-to-day basis. 
This hierarchy is used in the action model for PROJCON. Levels in the model are mapped to corresponding management levels to promote communication flow.

\section{ART Expert System Shell}

The Automated Reasoning Tool (ART) is an expert system shell with the following major components:

a. A language for knowledge representation using structured objects (schemata).

b. An inference engine.

c. Integration with the Lisp programming environment.

ART uses four main components (facts, schemata, rules and viewpoints) to represent an application's declarative knowledge. ART also has a compiler that maps the language into Lisp code.

Schemata or frames are used to describe declarative knowlege about a domain. Once compiled, schemata are represented as a data base of relations (termed facts by the ART documentation). For instance, the following schema definition: 
(defschema bw-1 "Concrete block wall number 1" (is-a cmu-wall)

(height-1 9)

(height-2 9)

(is-a structural-wall))

would be represented by the following relations (facts) after compilation:

$$
\begin{aligned}
& \text { (is-a bw-1 cmu-wall) } \\
& \text { (height-1 bw-1 9) } \\
& \text { (height-2 bw-1 9) } \\
& \text { (is-a bw-1 structural-wall) }
\end{aligned}
$$

The antecedents of rules (the conditions that must be matched before a rule can be selected) match data base facts (binary or otherwise) causing some change to the state of the system. The system state can be changed by asserting a fact into the database, retracting a fact from the database or calling a LISP function. The ART reasoning cycle begins with facts and rules in the initial state of the database and repeats a reasoning cycle until it reaches conclusions (no more rules can fire). Each reasoning cycle is composed of three stages:
a. Stage 1 - Match
b. Stage 2 - select
c. Stage 3 - Fire 
In stage 1, facts in the database are matched with patterns on the left-hand (antecedent) side of rules. Satisfied rules are placed on an agenda of rules to be fired. The agenda contains all activations of rules satisfied by stage 1. ART then selects the most important activation (based upon priority numbering called "salience"), thus designating it to be fired. "Firing" a rule consists of performing the action in its right-hand (consequent) side. This consequent action can simply be to print a message, perform a graphics routine or manipulate the database. Finally, the cycle is repeated until no more rules can fire.

only one rule activation can fire in each cycle. This means that some rules may be satisfied many times before they fire. Also, since the firing of one rule activation usually changes the database, some rules may initially be satisfied, placed on the agenda but never fire because their matches may be invalidated by a database change.

ART has debugging facilities that allow a programmer to specify limits (the number of rule firings), breakpoints, or to halt a program and freeze ART at any point during a run. Frame networks can be displayed 
through user modifiable windows. A source code file can be examined from an EMACS editor window. The user can move between the graphical frame depiction in an ART window to the EMACS editor window (with the cursor placed at the frame definition point in the file) with a mouse menu selection. EMACS allows incremental compilation of a frame definition as well as for LISP functions.

ART also has an icon editor that allows interactive graphical object definition which the system stores as a series of frames. These can then be saved to a file for later redisplay. Since ART runs in the symbolics Genera environment, new windows can be defined and modified easily.

Viewpoints fulfill the partitioning requirement for a frame-based system. They allow a database to be broken into smaller, more manageable sections, limiting rule pattern matching. Viewpoints can also be applied in the search process by having each partition act as a different search path. Viewpoints can be automatically created as new alternatives are found during search and destroyed if the alternative is disproved. 


\section{CHAPTER 2}

SYSTEM FEATURES

Two systems are discussed in this paper. PROJCON is a project control system that is the subject of a research grant from the Florida High Technology and Industry Council (grant awarded in December, 1987). CONPLAN serves as a test bed for the project planning and project scheduling portions of PROJCON.

\section{PROJCON}

PROJCON uses hierarchical model-based reasoning to simulate the analysis and decision processes used by a construction professional in three ways:

a. Generation of strategic plans and their execution as tactical plans in a manner in which construction planning is accomplished.

b. Creation of a schedule from a planning network

c. Interactive replanning/rescheduling, including changes in strategic plans.

To accomplish this, knowledge bases react to the contents and structure of hierarchical models and incrementally modify them. Knowledge bases are 
categorized based upon the functional aspects of construction management:

a. Strategic planning - high level management constraints

b. Project planning - create a graph showing intended installation order for each part.

c. Project scheduling - assign start dates to plan actions and evaluate resource, space constraints

d. Project chronicling and analysis - incorporate progress through interactive schedule updating

Hierarchical models are classified by information type:

a. Object model - describes the class of buildings that PROJCON will simulate.

b. Action model - shows methods utilized in order to construct each subobject in the object model.

c. State model - shows resources required to perform an action as well as optional actions when conditions change.

The system contains four subsystems, matching the functional aspects of construction management. First, the strategic plans are generated. Next, a planning network is generated for a particular building. A schedule is 
then created by linking the network into a period of time. Finally, a dynamic schedule-updating system allows schedule chronicling and analysis.

\section{Strategic Planning}

Strategic planning defines general organizational goals, tactical planning shows how the strategic plans will be implemented. For our case, we have a strategic planning subsystem that allows a user to identify portions of a building that should be accomplished with high priority. A user can also specify more vague requirements such as minimizing costs or activity durations or both. This subsystem allows strategic planning to occur dynamically, incorporating changes to strategic plans as job conditions warrant. It accomplishes strategic planning by creating rules for each strategic requirement defined by a user. The rules are defined so that they cause actions that could possitively affect tactical implementation of strategic plans to have higher priority when scheduling occurs. An evaluation function quantifies requirements relative to each other so that conflicting needs do not occur. 


\section{Project Planning}

Planning is accomplished by working from the top down, first identifying executive level actions and then elaborating details of how each are implemented. Interactions between detailed actions will be evaluated and a partially-ordered network graph is generated. This may (and many times does) affect executive-level tasks and changes will be reflected there. In this respect, the planning system first works from the top down and then reflects changes in a bottom up manner.

In more detail, the planning network is generated with the following steps:

a. A graphical interface is used to describe an instance of a building fitting in the class of buildings shown in the object model. Rule bases dynamically evaluate the building instance in the context of object model building characteristics, showing where deviations exist.

b. Once a building instance has been generated, a rule base creates an instance of the action model made up of a hierarchy of subnetwork graphs showing how to construct each subpart of the building instance. 


\section{Project Planning}

Planning is accomplished by working from the top down, first identifying executive level actions and then elaborating details of how each are implemented. Interactions between detailed actions will be evaluated and a partially-ordered network graph is generated. This may (and many times does) affect executive-level tasks and changes will be reflected there. In this respect, the planning system first works from the top down and then reflects changes in a bottom up manner.

In more detail, the planning network is generated with the following steps:

a. A graphical interface is used to describe an instance of a building fitting in the class of buildings shown in the object model. Rule bases dynamically evaluate the building instance in the context of object model building characteristics, showing where deviations exist.

b. Once a building instance has been generated, a rule base creates an instance of the action model made up of a hierarchy of subnetwork graphs showing how to construct each subpart of the building instance. 
c. Next, another rule base evaluates which detail actions cannot occur concurrently, partitioning the network into classes of actions that are capable of being scheduled simultaneously if given ample resources and workspace.

\section{Project Scheduling}

In the scheduling portion of this system, a state model is created showing resources required to implement each detail in the instantiated action model. A rule base evaluates each class of actions to ensure that the graph fulfills three requirements:

a. Available resources allow the plan to be implemented.

b. Available workspace will not hinder construction.

c. Tactical planning fulfills executive level strategic plans.

The graph will change based upon the above evaluations, and the planning rule bases are called upon to help alleviate detail action bottlenecks. One inadequacy with classical scheduling systems lies in the manner in which resource scheduling is accomplished. If many actions can occur simultaneously, activity priorities 
determine assignment of scarce resources. For those actions with identical priority, a random path through the actions is created by the scheduler. This occurs because no evaluation of the actions is made to determine a more efficient traversal. PROJCON will use constraint-directed search to find a better (shorter) path through these actions. Constraint directed search uses knowledge of constraints to limit the possible number of alternatives during search.

\section{Project Chronicling and Analysis}

A project chronicling and analysis mechanism allows interactive graphics for schedule updating and a rule base to help determine alternatives when project conditions change.

Through the use of interactive graphics, a user is able to update a schedule efficiently. A major portion of the chronicling and analysis mechanism of any project control system is dedicated to controlling the cost of the project. As such, the tracking system has been designed to aid in reporting current and future costs and to develop alternative budgets. 
When project conditions change (and they always do), classical project scheduling methods break down since they do not allow for alternatives in the network graph. Generally, the greatest benefits of project control are derived from the project planning portion of project control before construction begins. Once implementation begins, project control is relegated to the task of documenting what occurs because the network graph cannot be manually updated as quickly as field conditions change. The project chronicling and analysis subsystem contains a rule base that helps determine alternatives at any point in a project. Since the number of alternatives is combinatorially intractable, we are examining the possibility of using meta rules in a rule-writing system to combine meta rules into more complicated ones. In this way, the amount of knowledge that can be modeled is maximized and more alternatives can be evaluated.

\section{CONPLAN}

CONPLAN's primary goal is to perform the project planning portion of PROJCON. However, since the complete segregation of planning from scheduling is not possible, 
the project's last phase is dedicated to implementing portions of PROJCON's project scheduler.

CONPLAN performs its functions through the use of hierarchical knowledge representation, rule-based programming and domain specific problem solving strategies. Knowledge representation in the form of two hierarchical models stores plausible parts for a building and the methods used to construct each part. Rule-based programming was applied to create an interactive front-end for the program. Domain specific problem solving strategies are present in the categorization of knowledge within the models. The sections that follow will generalize each of these topics. The internal specifications give detailed implementation descriptions for each project phase.

\section{Model Hierarchy}

As shown in Figure 1 , the model hierarchy is subdivided into object, action and state models. The three submodels exist in parallel and are linked with structural relations at several points. Inheritance 
relations allow information to be shared within each model.

The object model contains organizational information showing the management hierarchy for the project; environment information describing concepts and objectives employed by the organizations involved in the project; physical information describing the building itself and the locale in which it is to be constructed.

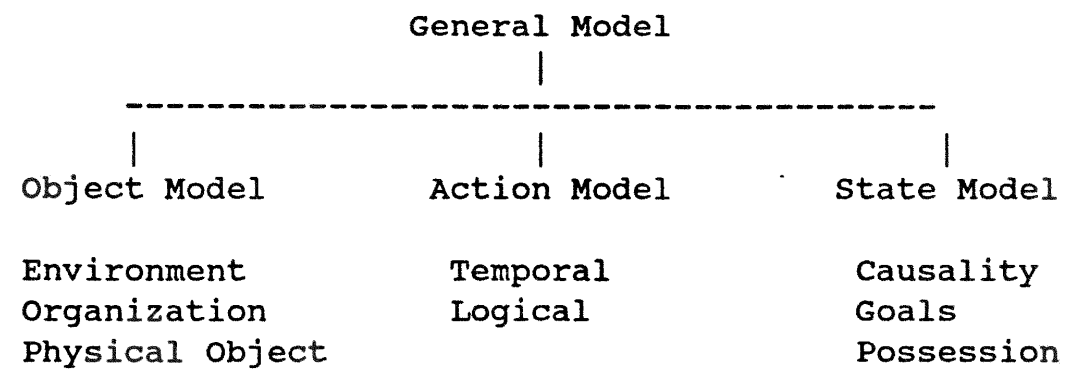

Figure 1 Model Hierarchy

organizational information describes the management hierarchy for a project. A construction project is subdivided into areas of specialization, with contract packages defined accordingly. A contract package is a conglomeration of work packages. The executive reporting level might be broken down by contract package. Competitive bidding determines which specialty contractor 
is awarded each contract package. These specialty contractors or subcontractors are responsible for completion of their defined scope. At the same time, subcontractors must work within guidelines developed and enforced by the construction manager. On large jobs, subcontractors often subdivide their scope of work, awarding subcontracts through competitive bidding. The depth of this hierarchy can create communicational interference and limit organizational effectiveness. The knowledge base should supply guidance to maximize information flow throughout the project.

Environmental information provides insights to the concepts and objectives employed by the organizations. Concepts tell what an organization intends to accomplish. Objectives signal the level of motivation that will be applied. Consolidation of these philosophies can highlight impending conflicts.

Physical information describes the building itself and the locale in which it is to be constructed. This involves a general building model, its parts and classification of subparts. Locale information includes 
resources available at the building location and local labor agreements.

Figure 2 shows sample schemata for organization model information. The organization hierarchy, member capabilities and ties to action and environment models are depicted.

(Defschema organization-aggregate

(is-a aggregate-schema)

(has-activities )

(has-vendors )

(has-subcontractors,

(has-supervisors )

(has-capability )

(subcontract-of ))

(Defschema organization-vendor

(is-a detail-schema )

(vendor-of )

(supplies-materials ))

Figure 2 organization Model Schemata

Schemata for the environment model are shown next, which include concepts and objectives. 
(Defschema environment-aggregate

(is-a aggregate-schema )

(has-concepts

(has-objectives )

(capability-of )\}

(Defschema environment-concept

(is-a detail-schema

(concept-of

)

)\}

(Defschema environment-objective

(is-a detail-schema )

(objective-of

)\}

Figure 3 Environment Model Schemata

The physical object model contains information about the building itself. Schemata for this model are shown in Figure 4. Relations in the physical object model show the hierarchy and orientation of building parts as well as building code requirements. 
(Defschema physical-object-aggregate

(is-a aggregate-schema

(has-parts

(above, below, next-to

(connected-to

(has-requirements

(has-activities

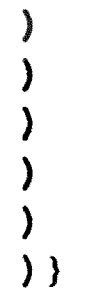

\{Defschema physical-object-part

(is-a detail-schema

(part-of

(has-attributes

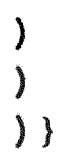

(Defschema physical-object-material

(is-a detail-schema

(material-for

(has-attributes

(supplied-by

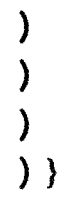

Figure 4 Physical Object Model Schemata

The action model stores information concerning time relations and activity hierarchy. Time relations are start/finish dates for each activity, activity durations and temporal relations. Start and finish dates are assigned by the system scheduler. Activity durations are user assigned and are constrained so that an allowable range is identified. Temporal relations define activity precedence as well as allowable parallel actions (see figure 5). 
(Defschema action-aggregate

(is-a aggregate-schema)

(before, after

(includes

(has-subact

(duration

)

(sum-cost

(Defschema action-detail

(is-a detail-schema )

(subact-of

(before, after

(includes

)

(duration

(cost

)

Figure 5 Action Model Schemata

The state model contains information concerning the current state of the world at each construction step. Project goals, causality, possession and composite states make up the state model. Project goals are represented as a hierarchy of either intermediate goals or project completion. on large projects, intermediate goals, called milestones, are used so that progress can be reported and evaluated. Causality defines the state necessary for an activity to begin. Resources required to perform an activity are identified via possession relations. composite states are subdivided into "and" and "or" states. An "and" state requires that all children be true 
or accomplished before that state is considered a candidate for selection. An "or" state requires only one of its children to be true or accomplished before it can be considered. Both types of composite states are shown in Figure 6 .

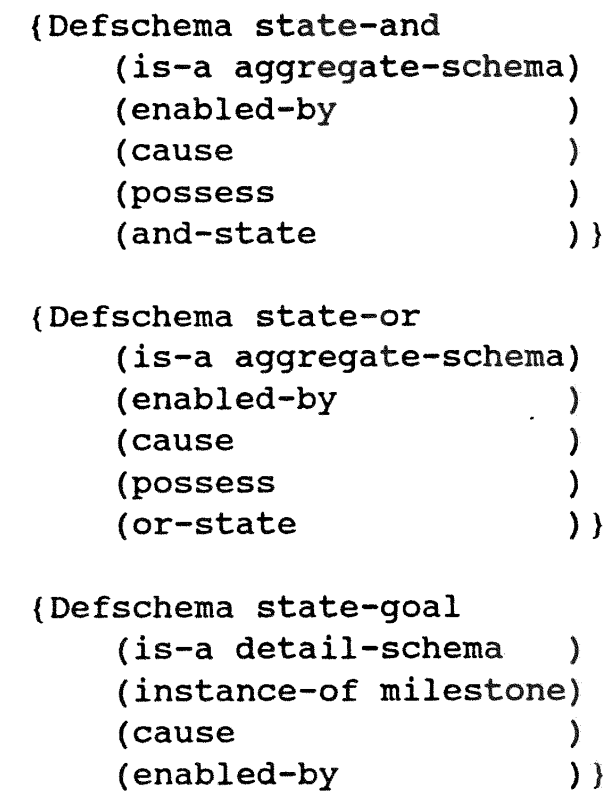

Figure 6 State Model Schemata

The state model is important only to the project scheduling portion of CONPLAN. As such, it did not receive as much implementational attention as the object and action models. 


\section{Phased Implemenation of CONPLAN}

CONPLAN was implemented in four phases which are described below. By breaking the project into independent sections, the need for one phase to be finalized before the next began was removed. The symbolics -3600 used to implement CONPLAN was configured with a minimum amount of resources making virtual memory limited. The virtual memory on a Symbolics Lisp Machine is obtained by creating a file on disk that is the same size as the desired virtual memory. If the disk size is limited (as it was in this case) the size of virtual memory is also limited, which can lead to execution limitations.

\section{Phase I - object Model}

In the first phase, the initial object model was developed and a rule-based user interface generated. The user interface allows development of a data base that describes a single story commercial building. The data base contains walls, foundations, windows, doors, roof and electrical circuits for the structure. The object model is also a data base that shows the possible parts of a structure that is built according to the South Florida 
Building code [37]. The user interface allows building part selection from those listed in the object model data base. It also contains rules that evaluate characteristics of the building as the user describes it. For instance, as a floor plan is developed, wall heights are input at each end (and interpolated linearly across the wall). A rule will fire if the heights are different at a point where two walls intersect (a corner).

The user interface follows an architect's process of designing a building by first elaborating the building structure followed by non-structural building parts. Structural parts for a typical building begin with walls, roof and finally the foundations. Foundations can be inferred from the building height (as specified in the South Florida Building code) and soil bearing characteristics. Nonstructural building parts such as finish materials, the electrical and comfort systems are specified after the building structure has been designed. A short description follows as to construction practices for structural and nonstructural building parts. The materials outlined in this paper for building parts are 
not all-inclusive. Other materials may be used, but the most prevalent are shown in this project.

Structural building parts support the building load above them. Foundations support walls, walls support the building roof. Foundations are constructed of concrete. Walls can be constructed of concrete block, cast-in-place concrete or wood. Roof structure can be of wood truss or bar-joist construction. Wood truss design is either gable or hip. Bar-joists are steel trusses that produce a flat roof.

Electrical cabling for commercial buildings must be as specified in the building code and the National Electric code [25]. These codes state specific requirements for commercial buildings based upon the use of each room in the building. As such, the number of outlets needed for a room (among other things) can be calculated based upon the length of walls within a room. The cable routing is not specified by the codes, but the maximum number of outlets per cable is limited. The heating and air conditioning systems (comfort systems) can be designed based upon each room's use. Heat loss (or gain) can be calculated for specific wall sections based 
upon the climate in which a building will be constructed. As is the case with most relatively simple structures, the building code restricts these calculations, limiting the number of possible outcomes.

The data base that describes a building must also show the spatial relationships of objects within the building. It should show that a roof is supported by the walls and the walls by the foundations. Also, as a floor plan is developed, rules create corner objects when two walls intersect. Once four corners have been generated, a room object is also created. Room objects are important to the resource evaluation system.

\section{Phase II - Action Model}

Once a floor plan has been generated, rules are applied that match the parts selected from the object model and call functions which create an instance of the action model for the particular building. This action model instance is made up of small subnets (less that 10 actions each) that show how each building part would be constructed if accomplished with no outside influences. Action model subnets are generated for individual 
instances of object model parts. If the general object model allows concrete block walls and the instantiated building contains four concrete block walls (bw-1 .. bw-4) then action model subnets are instantiated for each of the four walls. A mapping of abstraction levels within the action model to levels of management can be achieved by adding a management hierarchy to the object model. Relations could then show the action model level to management level mapping. This was not implemented in the current version of CONPLAN.

\section{Phase III - Partial ordering}

Next, a rule base evaluates the instantiated action model, adding an ordering relation between action model subnets. The ordering relation is only added when the construction order can be specified based upon building structure. The instantiated object model for a building shows spatial relationships between objects. These spatial relationships infer the manner in which some work must occur during construction of the building. For instance, if a structural wall is supporting a roof, then the structural portion of the wall must be constructed 
before the structural portions of the roof. The same can be said about foundations supporting walls. The output from this phase is an instantiated action model that has a partial ordering based upon required (and therefore not relaxable) construction constraints. Actions that show occuring simultaneously (those having the same start time) after this phase will be evaluated in the next phase for resource availability.

\section{Phase IV - Evaluation of Parallel Actions}

The final phase of CONPLAN evaluates actions shown occuring concurrently based upon available resources. Available resources include labor, material, building space and time. A simplified algorithm for this process involves:

a. Defining available resources through room size calculations and user prompts.

b. Performing a depth-first traversal of the instantiated action model, assigning depth values to actions. This assigns a start time to each action, representing the earliest possible start time. 
c. Fire rules that evaluate each group of concurrent actions (those with the same start time). When resources at a depth are all assigned, change remaining actions to the next lower level.

d. After all levels have been evaluated in this manner, check to see that time constraints (finish actions before a given time) are also satisfied. If not, relax constraints and try the search again. 


\section{CHAPTER 3}

\section{CONPLAN SYSTEM DESIGN}

\section{Rule-based Programming}

Rule-based programming is a way to encode the knowledge of an individual who is an expert in a particular field.

The following is an introduction to rule-based programming and includes a discussion of production rules and knowledge representation. An expert system consists of three major parts:

a. A set of rules

b. A database of known facts

c. An inference mechanism (or inference engine)

The inference engine controls the system, comparing the set of rules to the fact database and determining which rules could fire next. Only one rule out of the set of matched rules can fire, so the inference engine uses a conflict resolution strategy (there are many) to find it. Rules have two components:
a. An antecedent condition
b. A consequent 
The antecedent is a conjunction (or disjunction) of conditions that must be true before a rule can be considered for firing. The conditions are usually ternary relations, but are not limited to that category. Ternary relations are the minimum requirement when a frame-based representation is used for the fact database. The components of a ternary condition are a relation, an object and a characteristic. Figure 7 shows a fact with relation "has-parts", object "test-casel" and characteristic "roof". In this case, the object "testcasel" has a part called "roof".

(has-parts test-casel roof)

Figure 7 Sample Ternary Fact

In semantic net terms, a relation is a tie between two objects. The objects in this case are an object and a descriptive term for that object. The descriptive term (characteristic) is itself an object, with ties to other objects in the database. Within ART, when a relation is specified as part of a rule, it must be atomic (it cannot be a variable). However, an object and a characteristic 
may be a variable or an atom. The use of variables allows more general solutions (in a limited sense - not general in terms of general problem solver). More facts in the database are matched when variables are used in antecedent conditions.

The consequent of a rule may be a database assertion or a call to sequential program. The rule may also call for the deletion of an existing fact. Figure 8 shows a rule that retracts a fact, asserts a new fact and calls a lisp function. The rule, called "office-elect" has a priority (called salience in ART) shown by the variable "?*electrical*". Of all rules that have their antecedent conditions satisfied, the one with the highest priority will be the one chosen to fire. The second fact in the antecedent condition matches all rooms that have a "roomfunction" assigned. Next, a fact showing that the lighting load for the room has not been considered in building electrical calculations is matched and stored in a variable called "?matchl". A second fact showing the design lighting load for the room is matched and stored in variable "?match". The consequent condition first retracts the two facts stored in ?match and ?matchl and 
adds the lighting load for a single room to a total for the entire building. It then asserts a fact to prevent the same rule from firing again on the same room. Finally, a LISP function is called to perform some screen graphics.

(defrule office-elect

(declare (salience ?*electrical*)) (room-function ?room office) ?matchl <- (lights ?room no) ?match <- (lighting-load ?room ?load) $\Rightarrow$

(retract ?match 1 ) $\quad$;i; retract facts (retract ?match) (assert (lighting-load ?room $=($ ?load +15$))$ ) (assert (lights ?room yes)) $; ; ;$ assert new facts (display-room ?room)) ; ; ; a function call

Figure 8 Sample ART Rule

Knowledge representation is a method in which an analyst can categorize a problem, breaking it into smaller more manageable tasks. It allows a designer to organize information in a manner that can make large amounts of domain specific knowledge understandable.

\section{CONPLAN System Specifications}

CONPLAN (CONstruction PLANing) is the planning portion of PROJCON (PROJect CONtrol). CONPLAN is a 
combination of 2-Dimensional Computer Aided Design (CAD), hierarchical object representation and rule-based programming. Planning is accomplished in four phases. First, a user interactively specifies a "blueprint" for a one-story commercial building constructed under the building codes for South Florida [37]. He does so by selecting parts from a predefined set categorized in a general object model, thereby creating an instance of the object model. Next, an instance of the action model is created by selecting implementation methods from a general action model for each object chosen. ordering rules then apply a precedence relation to the instantiated action model subnets, tying the independent subnets together. Finally, the resulting graph is searched for a solution that fulfills various resource constraints.

\section{Functional Specifications}

The fundamental purpose behind CONPLAN is to show that a construction plan for a building can be inferred from a 2-dimensional building description. This is accomplished every day in the construction industry when engineers visualize a 3-dimensional image from 
2-dimensional blueprints and proceed to construct a building by following two general planning rules (or meta rules):

a. First construct the structural portions of the building, from the ground up.

b. Analyze constraints and plan remaining work so that as many constraints are satisfied as possible.

CONPLAN follows this format by:

a. Allowing the user to define a 2-dimensional building representation (a blueprint).

b. creating a 3-dimensional "visualization" by storing the spatial relationships in a database. This can be infered easily for our class of buildings since they are only one story. It is a much more difficult problem infering spatial relationships for multistory buildings.

c. Determining methods to construct each part of the building as if they were independent of each other.

d. Applying ordering rules that infer order of actions from the structure of the building (stored in the database). 
e. Searching the resulting graph to find a result that satisfies constraints defined for the project.

The system specifications are subdivided into two categories: external specifications and internal specifications. External specifications describe the user interface for the software to be developed and show all screen formats with which the user will interact. Internal specifications describe the data structures used to implement each the program module.

\section{Phase 1 External Specifications}

Phase $I$ is an interactive graphical user interface that allows a user to create a floorplan for a one-story commercial building constructed according to building requirements local to south Florida. The building can be constructed of concrete, concrete block or wood. All structural elements such as foundations, slabs, walls and roof will be defined in phase 1. Non-structural walls as well as doors, windows, room functions and electrical circuits will also be defined for the building through interactive menu selections. 
Figure 9 shows the initial screen that will be displayed when CONPLAN is activated. Four windows will open for commands, interactive graphics, menu selections and a firing rule agenda. 


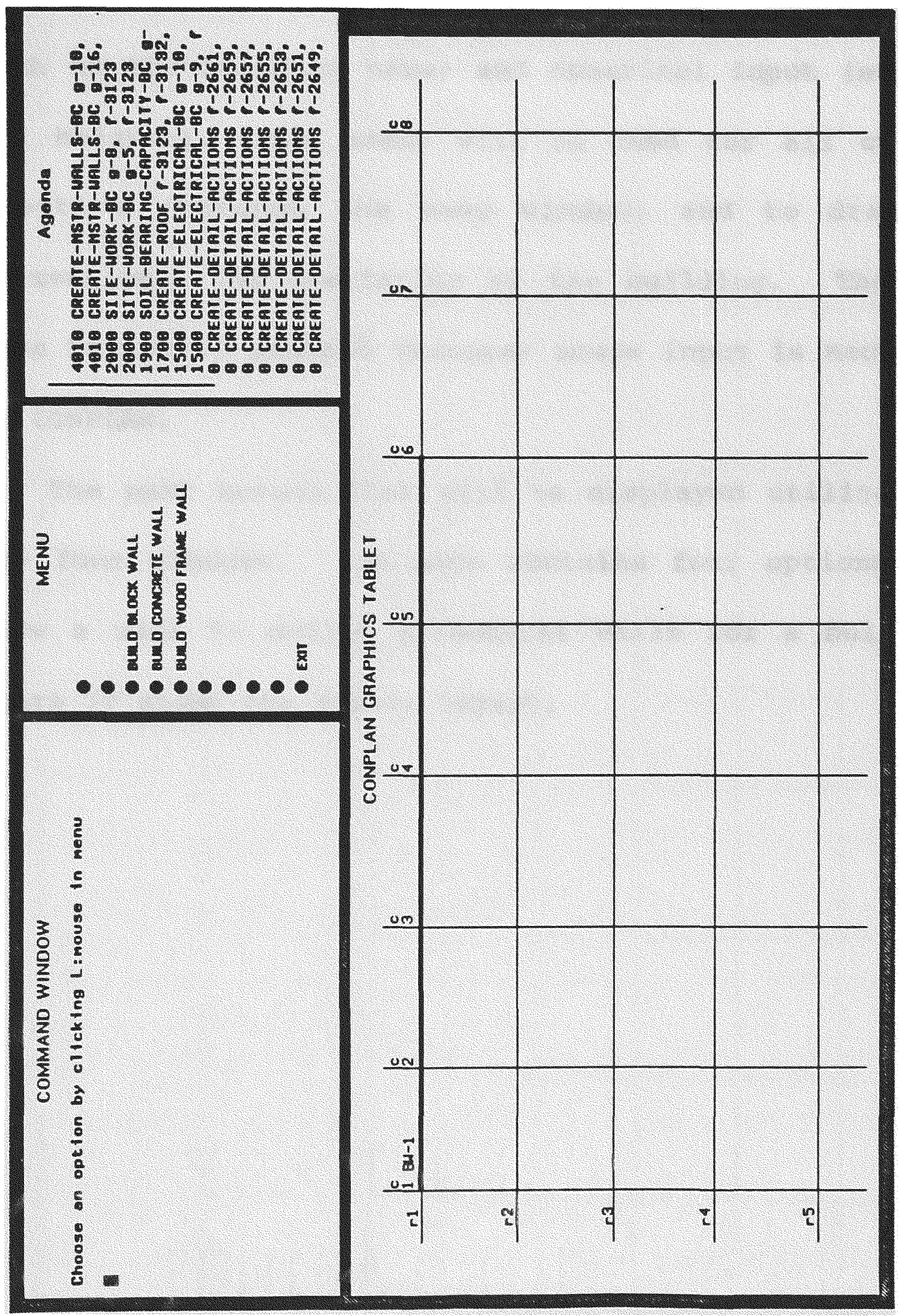

Figure 9 CONPLAN Initial screen 
All keyboard input will be through the command window (upper left) and will be limited to a few object names (such as the building name) and numerical input (such as wall heights). The mouse will be used for all command selection (through the menu window) and to draw the 2-dimensional representation of the building. The left mouse button is pressed whenever mouse input is necessary for CONPLAN.

The next screen that will be displayed utilizes the same four windows. The menu contains four options that allow a user to define structural walls for a building. Figure 10 shows the screen layout. 


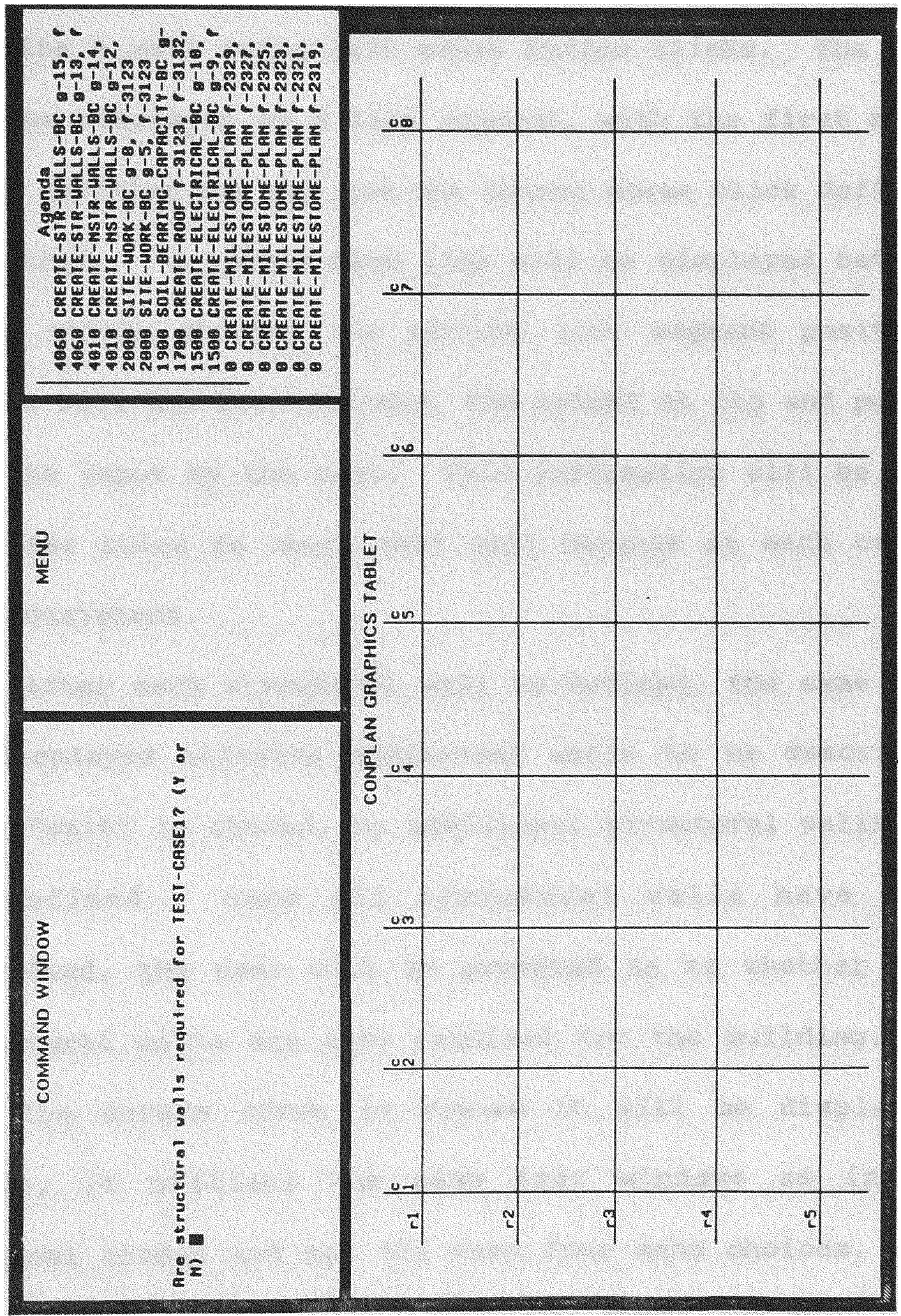

Figure 10 Screen Layout For Structural Wall Selection 
"Build Block wall", "Build Concrete Wall" and "Build Wood Frame Wall" options cause the user to be prompted to describe a wall using left mouse button clicks. The wall will be displayed as a line segment, with the first mouse click defining one end and the second mouse click defining the other. A rubber-band line will be displayed between mouse clicks showing the current line segment position. Once a wall has been defined, the height at its end points will be input by the user. This information will be used by later rules to check that wall heights at each corner are consistent.

After each structural wall is defined, the same menu is displayed allowing additional walls to be described. once "exit" is chosen, no additional structural walls can be defined. Once all structural walls have been described, the user will be prompted as to whether nonstructural walls are also required for the building. If so, the screen shown in Figure 10 will be displayed. Again, it utilizes the same four windows as in the original screen and has the same four menu choices. The new menu has five choices to create doors, windows, nonstructural walls and to exit this screen. 
Once the "Door" option is chosen, a new menu will be displayed showing all possible doors that could be used in the building. Using the left mouse button, the user can choose a door type and then show its location and width on the blueprint in a manner similar to that used when describing a wall. Once complete, the non-structural wall menu will again be displayed.

An identical method will be used to create windows as was used for doors. A menu of all possible window types will be displayed, allowing selection by the user. The location and window width can be defined with left mouse button clicks. Once complete, the non-structural wall menu will again be displayed.

Nonstructural walls are described in an identical manner as that used for structural walls. Their database representation differs from structural walls in that they have an attribute "non-structural" assigned to them. This characteristic will be used in the next project phase to help infer the order of actions by placing emphasis on structural actions before nonstructural actions.

The next screen will prompt the user for information as to the use of each room in the building. Rooms are 
defined as parallelograms made up of four corners, with each corner connecting two differing walls. At this point, rooms have been determined and the screen shown in Figure 11 is used to define the function of each room. Although not used in the current version of CONPLAN, this information is important to help calculate lighting, heating and cooling requirements for the building. 


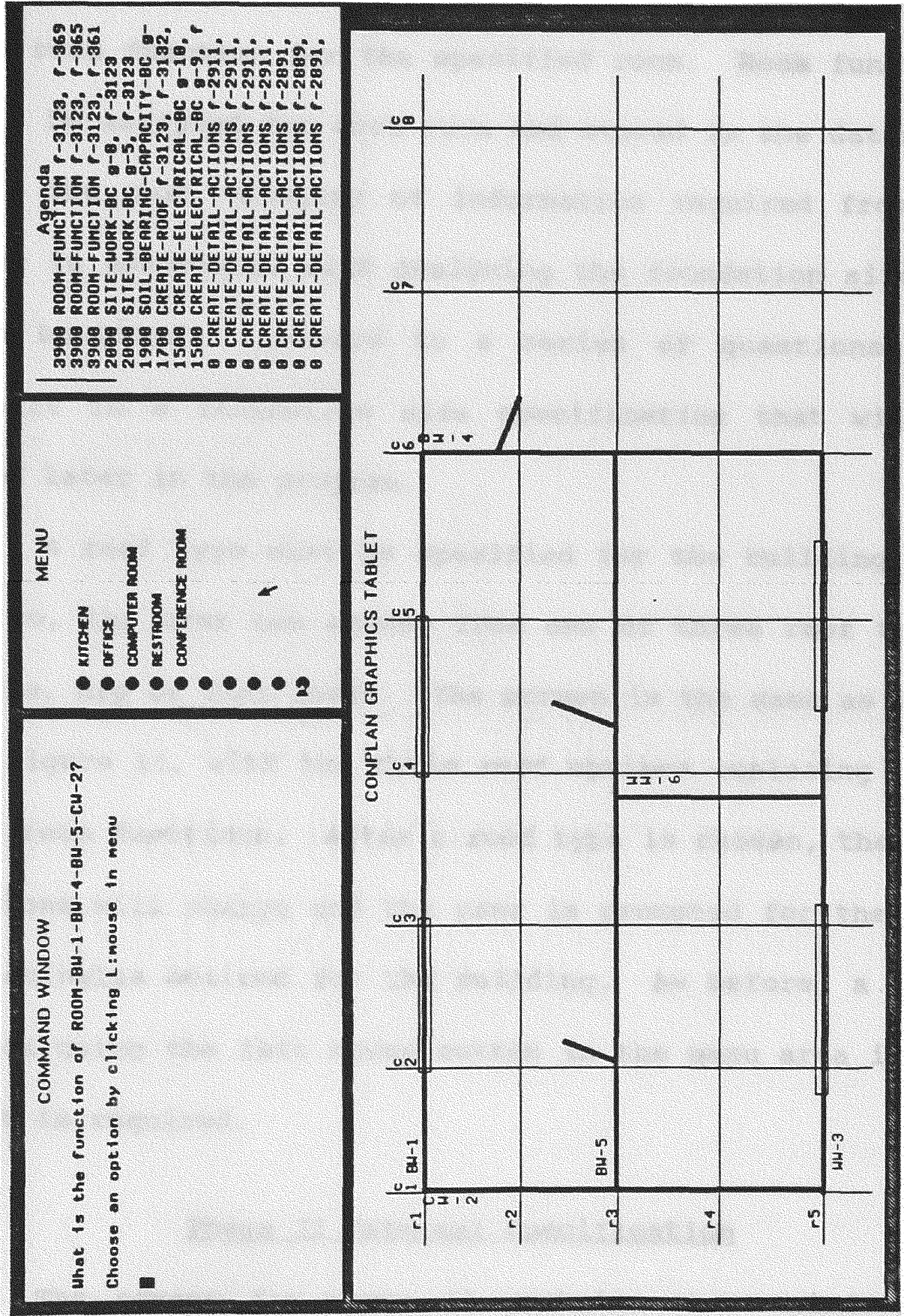

Figure 11 Screen Used For Room Function Selection 
There are five room functions shown in the menu. The user will be prompted (as shown in Figure 11) to choose the room function for the specified room. Room functions will be assigned for each room and stored in the database. The next category of information required from the user is associated with designing the foundation size for the building. Answers to a series of questions will result in a foundation size specification that will be used later in the program.

A roof type must be specified for the building. To do so, the user can choose from one of three roof types: gable, hip or flat roofs. The screen is the same as shown in Figure 11, with the three roof options replacing those for room functions. After a roof type is chosen, the menu options will change and the user is prompted for the type of shingles desired for the building. As before, a mouse click using the left mouse button in the menu area is all that is required.

\section{Phase II External Specification}

The screen for phase II contains three windows as shown in the following figure. 


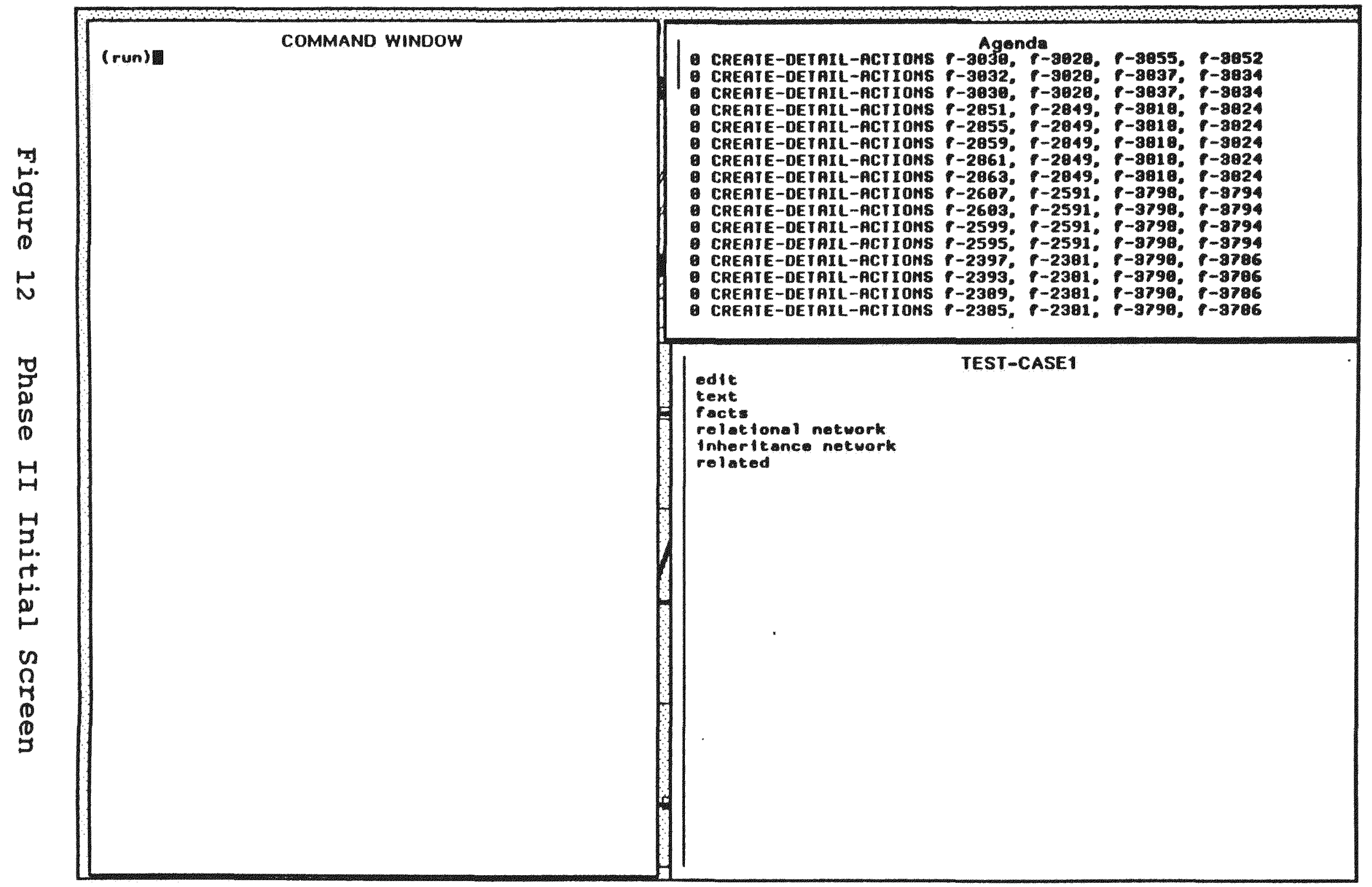


The command window shows the results of each rule firing. The agenda window shows the rule conflict set, with the rule shown on top having been chosen to fire next. The third window (labeled TEST-CASEl in figure 12) is the ART expert system shell command menu.

The command window is a IISP interpreter window allowing direct access to all defined functions. The only action required of the user for this phase is to begin exectution by entering the "run" command in the command window. The user can watch as objects are created, added to the database and displayed on the command window. When a rule completes execution, conflict resolution determines the next rule and more objects are generated, stored and displayed. This cycle continues until no further rules can fire, ending phase two.

\section{Phase III External Specification}

The external specification for this phase is identical to that for its predecessor. Once phase 3 is loaded, the only user input is to invoke the system by entering "run" within the command window. Then watch as 
rules fire, evaluating the order of actions for differing objects.

\section{Phase IV External Specification}

The screen layout for phase IV is identical to that of previous phases. The user can invoke this phase by entering "run" in the command window. User input is required to define resource availability constraints. The user will be prompted for these values as shown in Figure 13. 


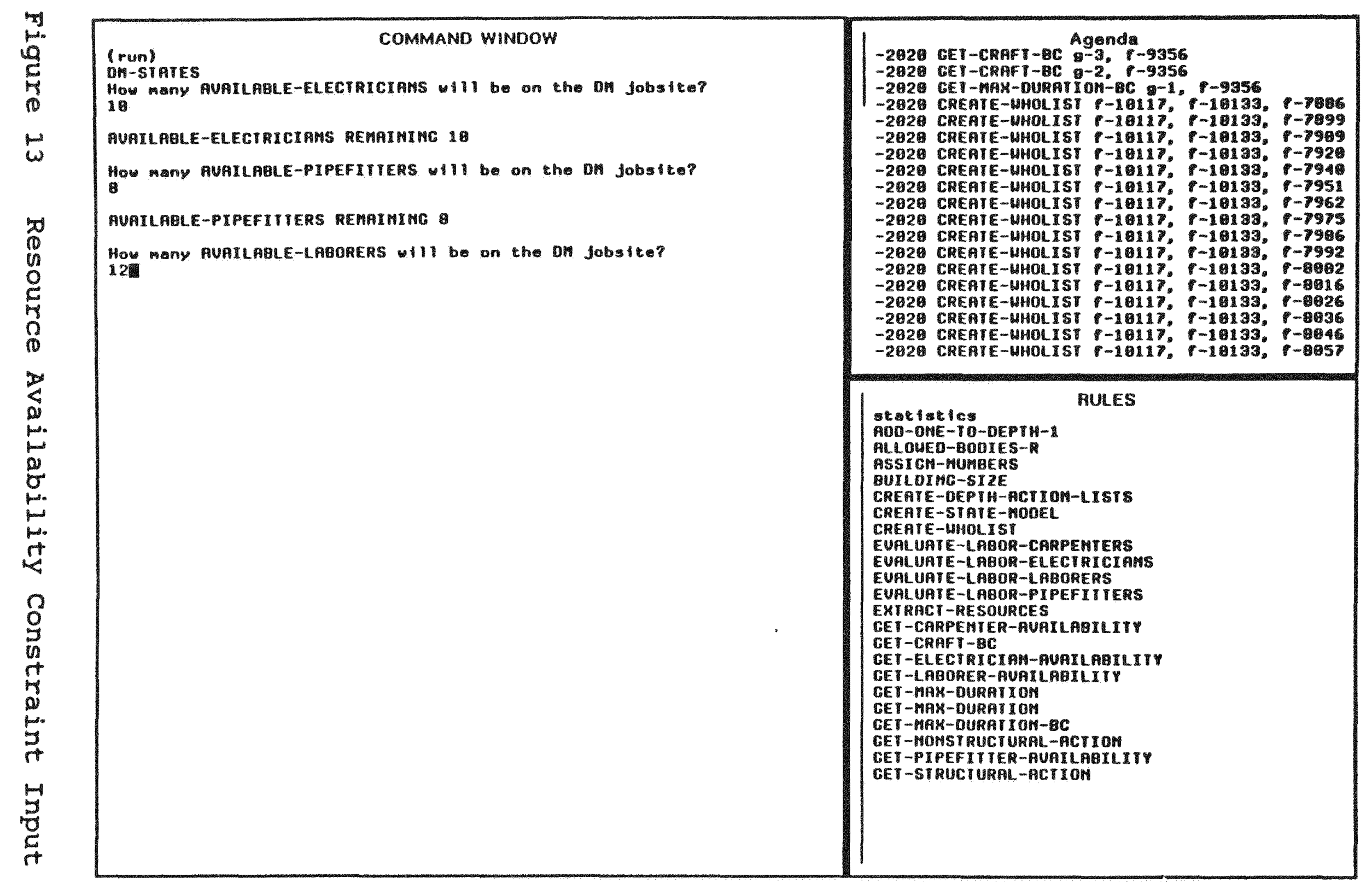


With this input, the system will begin to fire rules that create a list of all actions to be evaluated, perform a depth-first traversal of this graph and assign resources to each action. If an acceptable solution can not be determined within the given constraints, one or more constraints will have to be relaxed. User input is required to relax constraints by selecting those that can be changed in some manner (add more labor, increase time, etc). These inputs will be done through prompts caused by backward chaining rules and will present the user with constraint relaxation alternatives. The user can choose one constraint to relax and the system will attempt to solve the problem again. When a solution has been found, no more rules will fire and the system will halt.

\section{General Model Internal Specifications}

Internal specifications describe data structures internal to the program. They also describe actions that the user will not see and does not need to be concerned with. All rules developed in this project will be outlined in succeeding sections as well as their effects on the model hierarchies. 
The database, before the user interface rules fire, will be classified into two models: general object model and general action model. The general object model stores information about possible configurations that a building design could have. Possible parts for a one-story commercial building (within the limited domain of this project) are defined in the general object model. The general action model contains information about how to construct each part shown in the general object model. These subplans are based upon independent construction of each part and do not consider such constraints as limitations on labor, material, equipment and space.

The general object model is a hierarchy of objects that are at different levels of abstraction. At the root is the building model level that has categories below it in a tree-like fashion. The second level contains category descriptions for different classes of parts within a building. The third level is more specific (less abstract) and further partitions a category into subcategories. The relation "has-categories" is used for this part of the hierarchy and allows inheritance of 
characteristics from parents to children. Figure 14 shows this hierarchy for the general object model. 


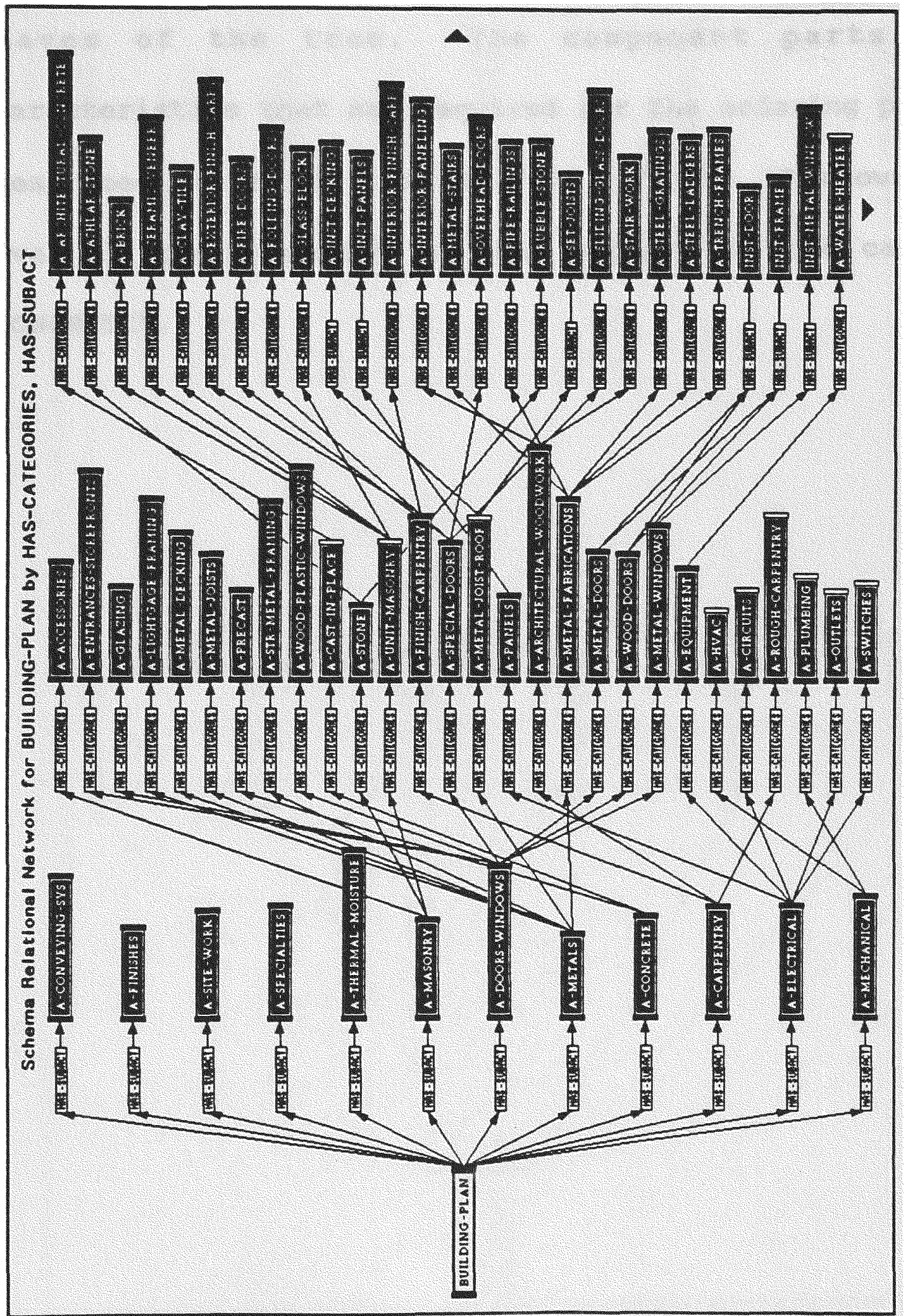

Figure 14 General Object Model Hierarchy 
At the fifth level in this hierarchy, objects are subdivided into their component parts, which comprise the leaves of the tree. The component parts have characteristics that are required for the ordering process (described later in this thesis). Figure 15 shows this level in the hierarchy for one segment of the category "CONCRETE" . 


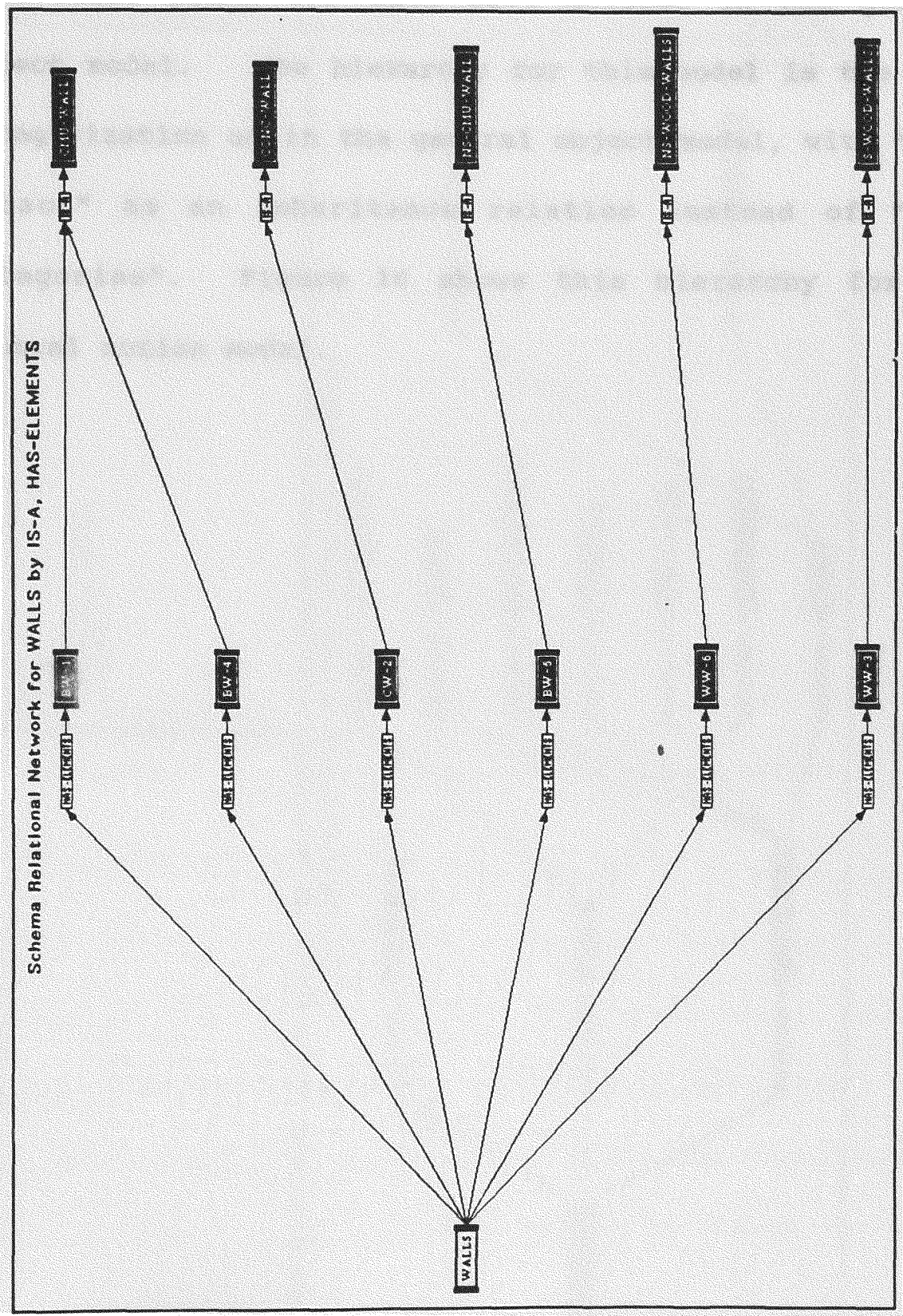

Figure 15 One Category From Object Model 
As stated earlier, the general action model contains individual plans for each part defined in the general object model. The hierarchy for this model is the same categorization as in the general object model, with "hassubact" as an inheritance relation instead of "hascategories". Figure 16 shows this hierarchy for the general action model. 


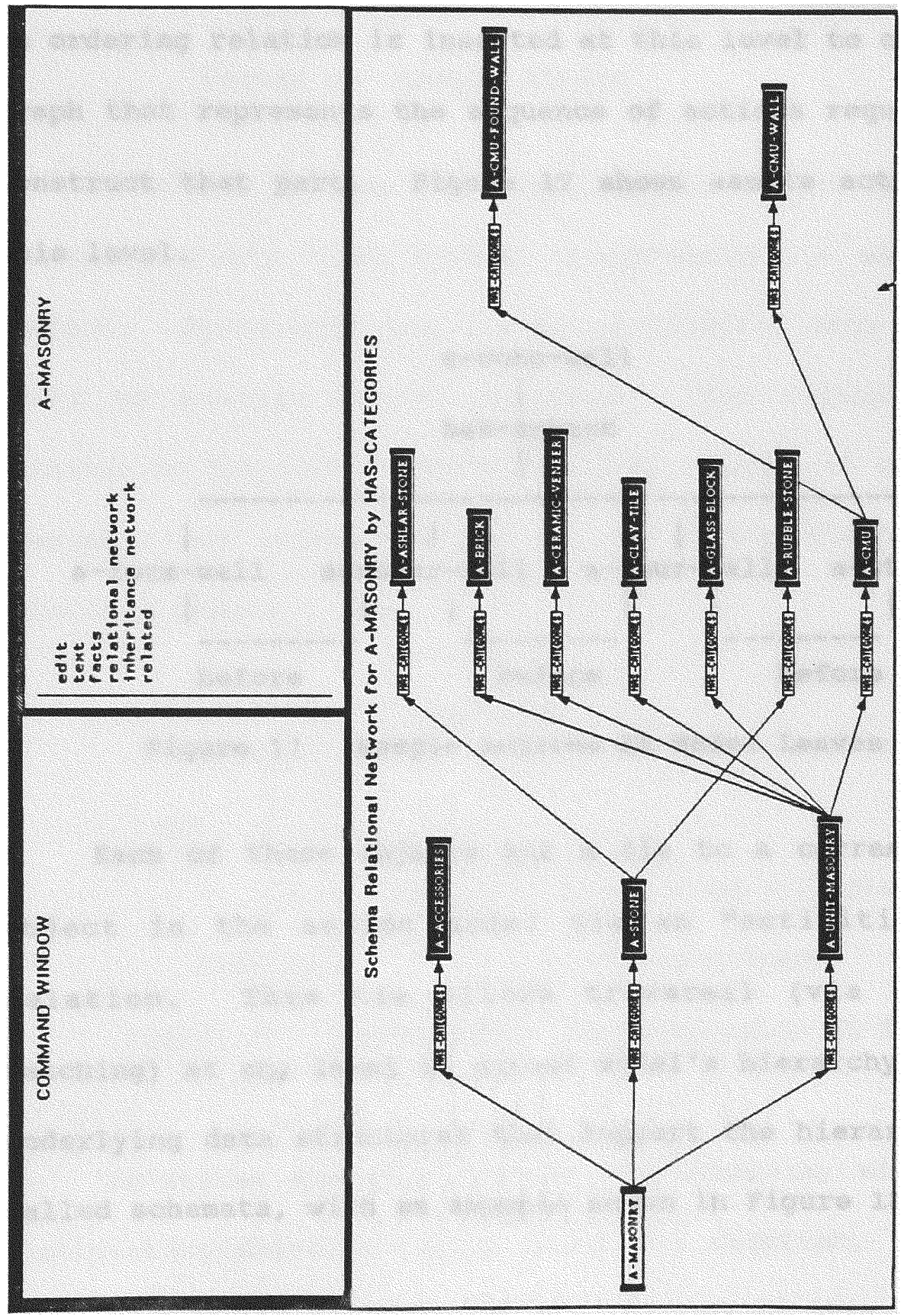

Figure 16 Action Model Hierarchy 
The lowest level in this model delineates the actions required to build each part in the general object model. An ordering relation is inserted at this level to create a graph that represents the sequence of actions required to construct that part. Figure 17 shows sample actions at this level.

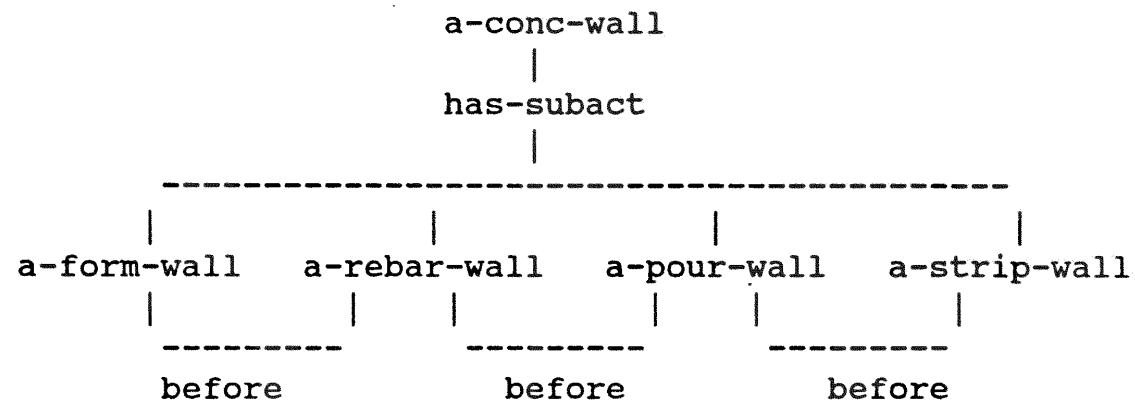

Figure 17 Sample Actions At Model Leaves

Each of these objects has a tie to a corresponding object in the action model via an "activities-for" relation. This tie allows traversal (via pattern matching) at any level in either model's hierarchy. The underlying data structures that support the hierarchy are called schemata, with an example shown in Figure 18. 
(Defschema a-concrete

(activities-for concrete)

(before-ms bldg-dried-in)

(has-categories a-cast-in-place a-precast))

Figure 18 Sample Schema

A schema defines an object, has a unique name, and contains slots that define characteristics about the object. Slots have two fields, a relation name and the name of another object or value. The relation is a pointer to the other object or value. Figure 19 shows the semantic net representation of the schema shown in the previous figure.

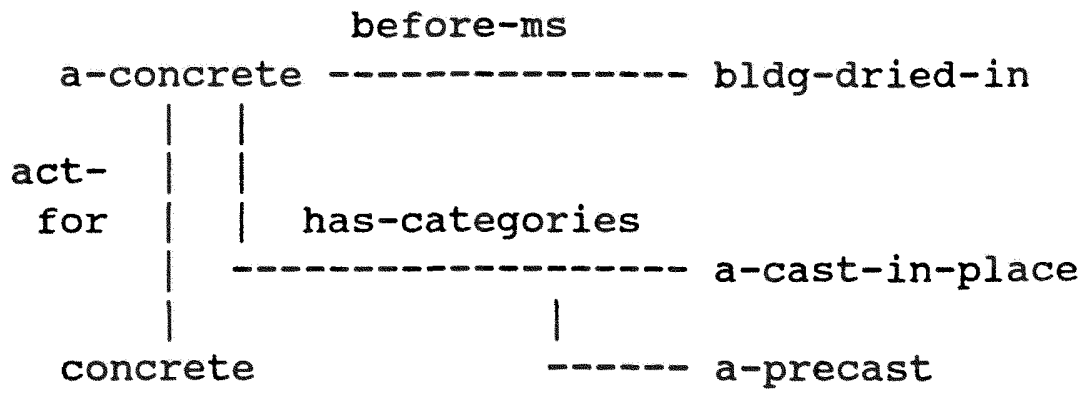

Figure 19 Semantic Net Representation of Schema

Relations are also defined via schemata, with slots delineating characteristics about the relation. In ART, there are seven kernel schemata that serve this purpose. 
Figure 20 shows the definition schema for one of the relations displayed in Figure 19.

(Defschema has-categories

(instance-of relation)

(element-of relations)

(inverse category-for)

(slot-how definite)

(slot-what nothing)

(slot-how-many multiple-values)

(slot-multiple prompt))

(Defschema category-for

(instance-of inh-relation)

(element-of inh-relation)

(slot-how definite)

(slot-what nothing)

(slot-how-many multiple-values)

(slot-multiple prompt))

Figure 20 Sample Relation Definition

The two relations shown in the example are inverses of each other, which is why both are shown. The relation definitions shown in Figure 20 contain four of the seven kernel schemata (all slots that begin "slot-" in the example). The seven kernel schemata are:

a. Slot-what: Tells whether the object or value pointed to by this relation can be shared with other objects that are tied via inheritance relations. 
b. Slot-how-many: Defines how many values can be stored in this slot for each object (either single-value or multiple-values).

c. Slot-multiple: Defines what action to take if more than one value is placed in a single-value slot (as defined in slot-how-many).

d. Slot-how: I did not use this relation to its fullest extent (therefore I do not have an indepth knowledge of its definition). It defines how information is shared when hypothetical reasoning is used within an ART program.

e. Slot-input: Defines input strings that will be accepted from the keyboard for this slot name.

f. Slot-output: Defines output strings that will be displayed when this slot is shown to the user.

g. Slot-input-output: Defines a combination of the two previous input and output formats.

Relations are one of two types: inheritance relations or non-inheritance relations. The relation defined in Figure 20 is shown as two inverse relations. The first, "has-categories", is a non-inheritance relation as specified by the "instance-of" and "element-of" slots. 
The second relation, "category-for" is defined as an inheritance relation as shown by the contents of the same two slots. Inheritance is an important characteristic of knowledge representation (as opposed to relational databases) in that it allows information that is known at one object in a network to be also known at others. Characteristics can be shared between related objects in an automatic fashion.

Two programmer defined attributes must be set before inheritance can occur. First, an inheritance relation must be defined and applied between parent and child objects. Second, a non-inheritance relation must also be employed that identifies a characteristic of the parent object. If the non-inheritance relation has the "slotwhat" kernel schema defined as either "share-value" or "copy-value" then the child will inherit that characteristic from the parent.

Different results will occur if the non-inheritance relation's "slot-what" value is "share-value" or "copyvalue". Share-value adds a pointer from the receiving object to the characteristic and both objects "share" the same characteristic. Copy-value does not behave in this 
manner. A copy of the characteristic is made, given a unique name, and pointed to by the receiving object. Figure 21 shows this differentiation as well as the properties required for inheritance.

The basic difference between non-inheritance and inheritance relations is that non-inheritance relations describe characteristics about an object while inheritance relations allow those characteristics to be shared with other objects.

Relation definition using share-value:

(Defschema before-ms

(intance-of relation)

(slot-what share-value))

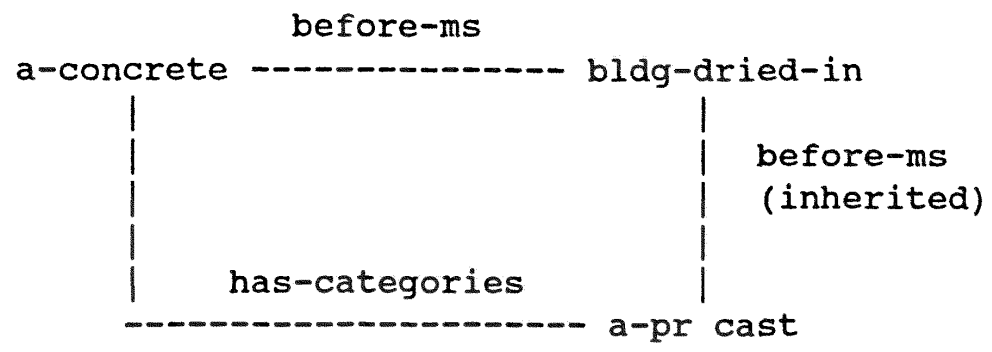

Figure 21A Inheritance Properties Using Share-Value 
manner. A copy of the characteristic is made, given a unique name, and pointed to by the receiving object. Figure 21 shows this differentiation as well as the properties required for inheritance.

The basic difference between non-inheritance and inheritance relations is that non-inheritance relations describe characteristics about an object while inheritance relations allow those characteristics to be shared with other objects.

Relation definition using share-value:

(Defschema before-ms

(intance-of relation)

(slot-what share-value))

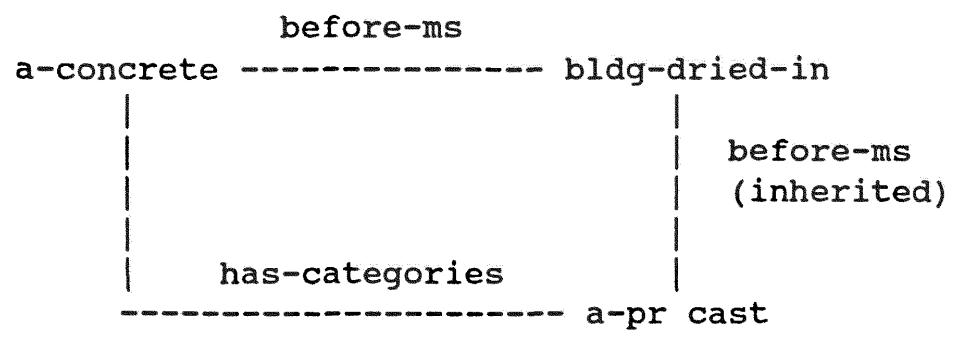

Figure 21A Inheritance Properties Using Share-Value 


\section{Phase I Internal Specification}

Data gleaned through the user interface is stored in a hierarchy of database objects. The foundations, walls, roof, doors, windows and electrical circuits, as defined by the user, are stored as an instance of the general object model. A name is assigned to the object model instance (see external specification) and stored as a hierarchy using the same data structures defined for the general models. Figure 22 shows this hierarchy for objects defined in an object model instance called TESTCASE1. 


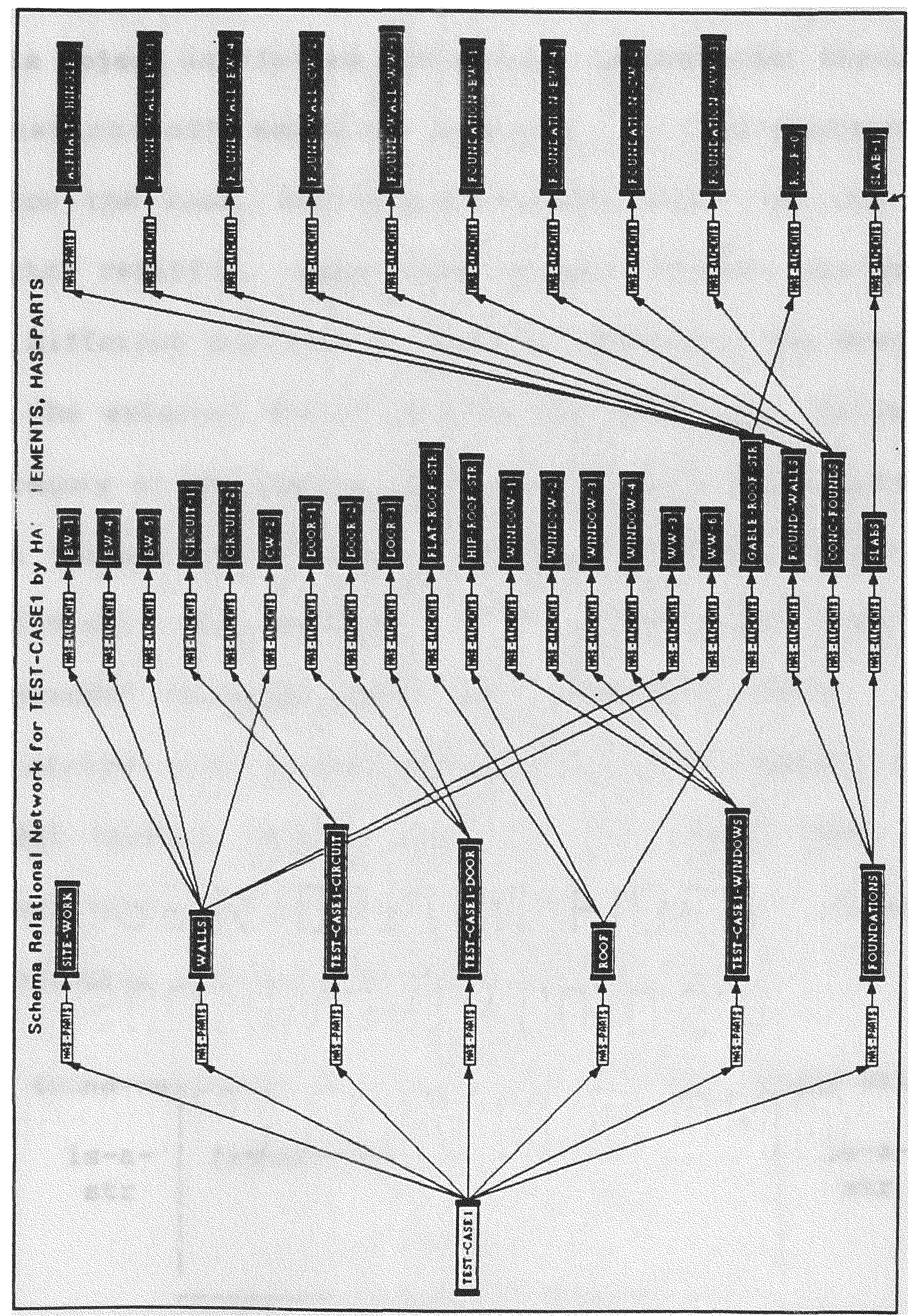

Figure 22 Object Model Instance TEST-CASE1 
The root of this hierarchy is an object whose name corresponds with the building name input by the user. This object is tied to the general object model through an "instance-of" relation (inverse of "has-instances"). Below the root, building parts are shown via the "hasparts" relation. Each building part category was created by different portions of the user interface (as described in the external specifications for phase 1). Below each category at the second level are instances of objects from the general object model that have been chosen for this building. For example, under "found-walls" the "haselements" relation shows four foundation walls. These foundation wall instances are tied to the general object model through "is-a" relations (not shown) that allow inheritance of structural characteristics. Figure 23 shows this relationship for foundation walls.

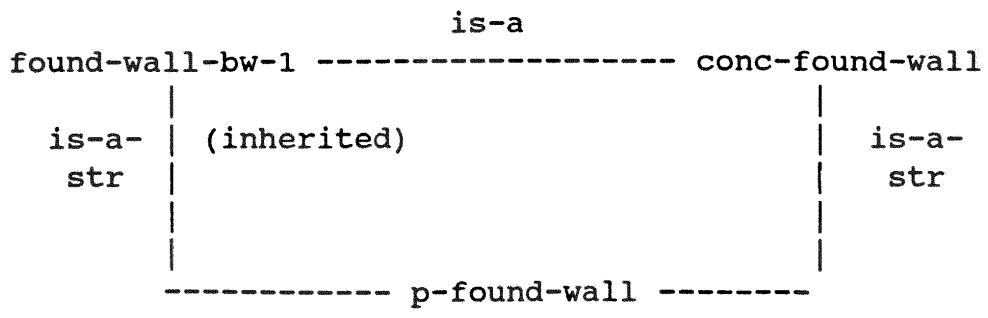

Figure 23 Explicit and Inherited Model Ties 
The hierarchy for an instance of the general object model is shown in Figure 24. It is taken from one test case used for this project.

Figure 25 shows the wall category for TEST-CASEl and displays six walls with their ties to the general object model through "is-a" inheritance relations. 


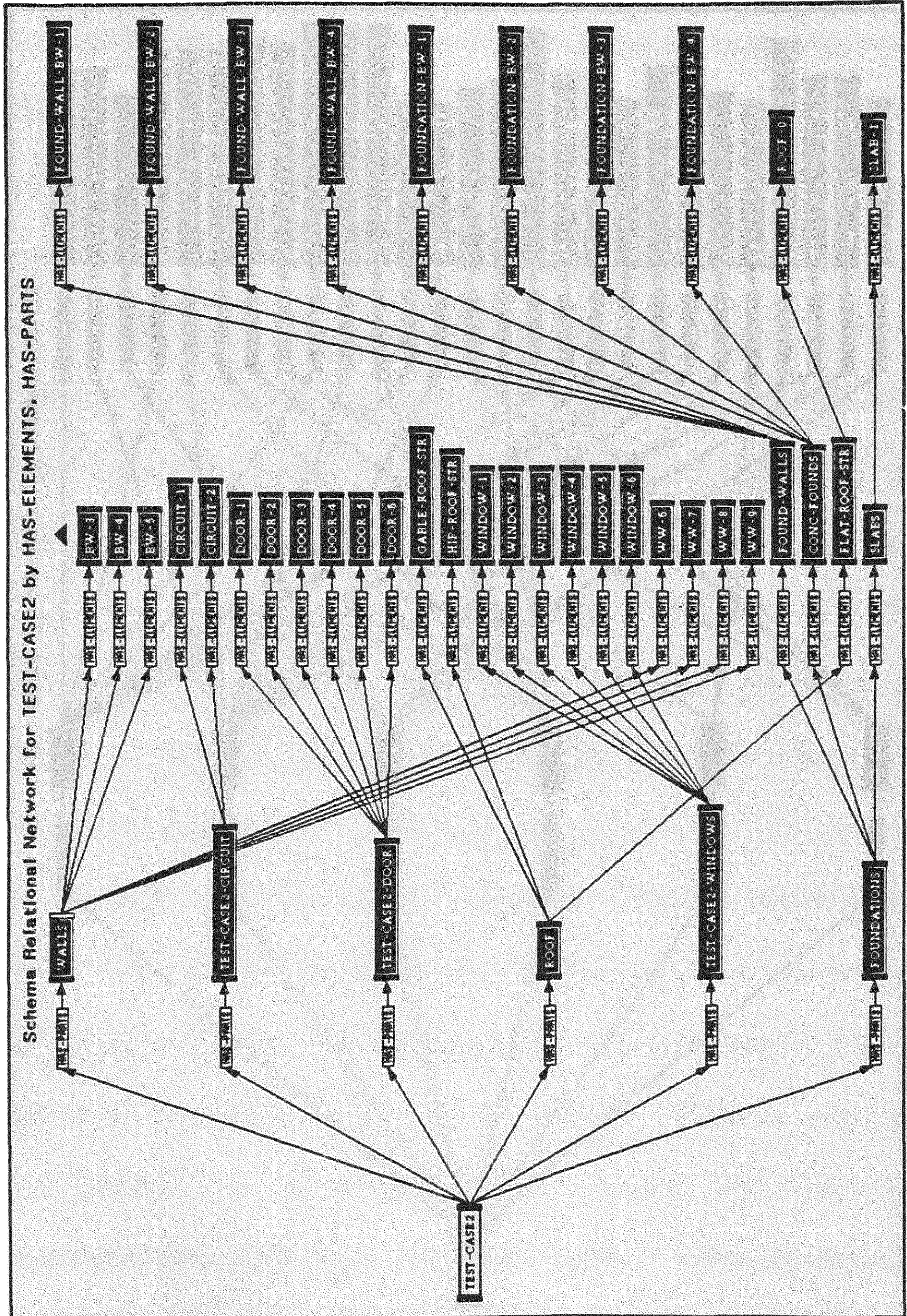

Figure 24 Object Model Instance For TEST-CASE2 


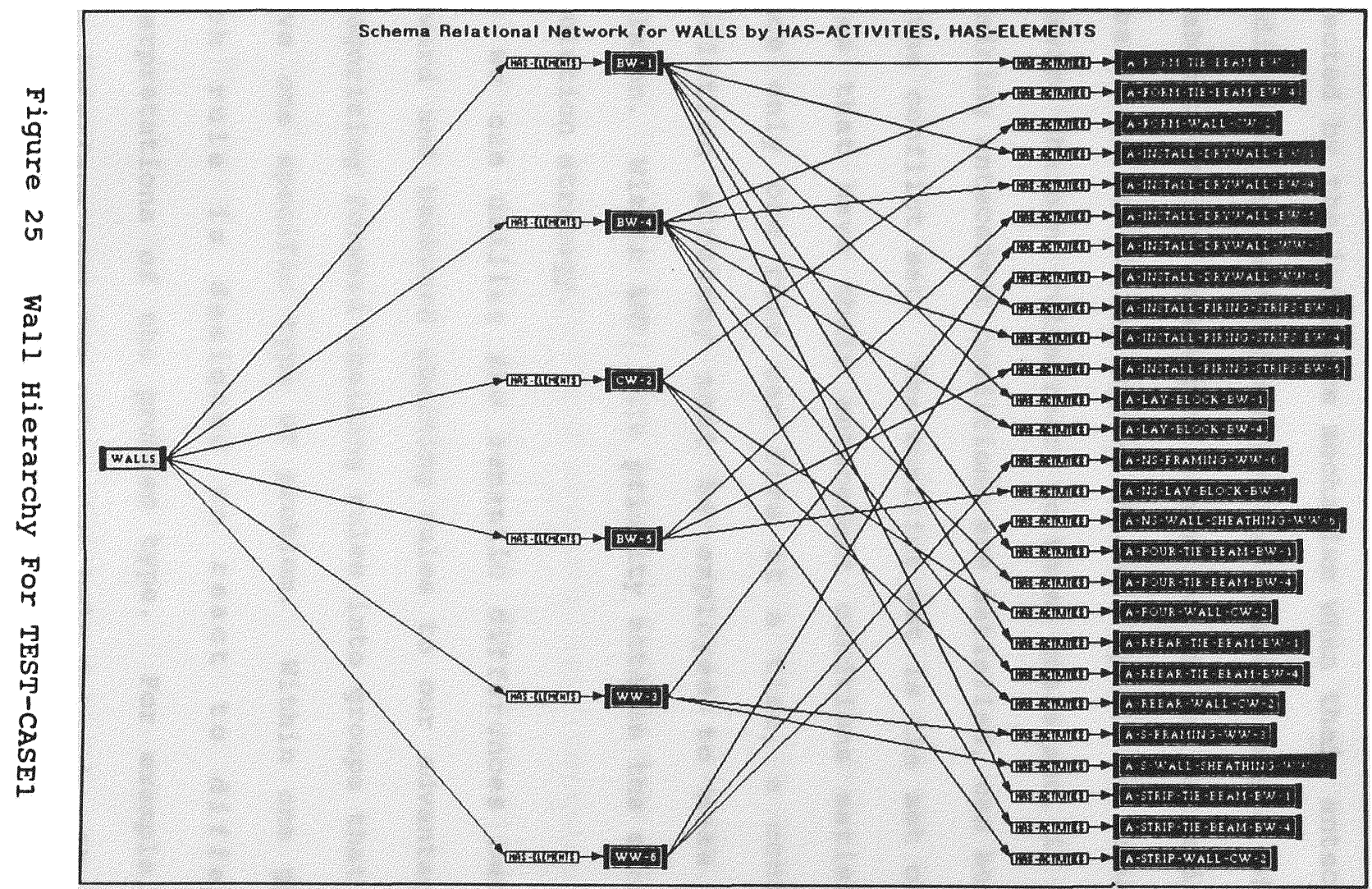


Forward and backward chaining rules are used to enact all database changes. Forward chaining rules can be selected by the inference mechanism when their antecedent conditions are satisfied by the current state of the database. If an antecedent condition can be satisfied by a backward chaining rule, then a goal is created. Backward chaining rules react to these goals and, if their remaining antecedent conditions are satisfied, can be part of the conflict set. The conflict set is the set of all rules that have their antecedent condtions satisfied. Since only one rule can fire at a time, a conflict resolution strategy must be employed to make that decision. Within ART, rule priority acts as the conflict resolution strategy.

We can utilize the semantic differences between forward and backward chaining rules to our advantage by categorizing forward chaining rules into groups that each solve one specific type of problem. Within one group, each rule is designed to react to different interpretations of the problem type. For example, the user interface contains rules that help determine the type of subsurface exploration desired for a building site. 
Three rules are designed to handle this problem (although many more would be required for the general case). Two are forward chaining rules designed to react when a specific type of subsurface exploration is determined and one is backward chaining.

Before the forward chaining rules are able to react, the inference mechanism checks to see if any backward chaining rules could assist by solving goal states. If such rules are found (one in this case), goals are created by the inference mechanism. The backward chaining rule attempts to resolve the goals by performing its programmer defined consequent. In my case, backward chaining rules are used to prompt the user for goal solving data.

This forward/backward chaining rule technique was used throughout this project. In the first phase, the following categorization exists:
a. Structural walls
b. Nonstructural walls
c. Electrical
d. Foundations
e. Doors
f. Windows 
g. Room calculations

h. Site work

i. Roof type

Backward chaining rules serve as a mechanism to extract data from the user to guide the system in selecting forward chaining rules for the conflict set. In some cases, a category may initially only solve one problem type, but could be elaborated for a more general solution. In other cases, backward chaining rules are not employed at all. In such cases, rule priority (along with current database conditions) allowed access into the conflict set. I will elaborate on these methods in the following paragraphs.

Structural walls, nonstructural walls, electrical and foundations were categories that I limited to one problem type each, with a backward chaining rule to prompt the user for data. By one problem type, I mean that for the category "structural walls", only one type of structural element may be used, namely structural walls. A more general case would also have to consider structural columns and beams. Three categories (with the exception of foundations) use a "yes" or "no" prompt as the backward 
chaining user input. The foundations category also requires soil bearing capacity to be determined.

Doors and windows do not use backward chaining rules to invoke them. They will react, once a building instance has been created, when all rules having higher priority have had an opportunity to do so. Room calculations include four rules that:

a. Create corner objects

b. Create room objects from corners

c. Evalutate wall heights at corners

d. Allow the user to choose room function

Corner objects are created when end-points from two walls overlap at a screen coordinate. Rooms are created when four corners connect four differing walls. Wall height is input by the user when the wall is initially described. Wall height at corners is checked for consistency by one rule. Consistency checking is an area that could be elaborated in further versions of this system. As with the wall height rule, the building design could be further evaluated by the system in a manner consistent with methods used by a design professional. 
site work and roof type employ the rule categorization method, using forward and backward chaining rules. The method was described earlier using site work as an example. Roof type selection uses the same method of elaborating different problem types within a problem category and using a backward chaining rule to allow user input to select the problem type. In this case, three roof types are available (but many more could be added) and one backward chaining rule gets user response.

\section{Phase II Internal Specification}

Detail actions created in phase II are stored as new leaves in the general action model. They are also tied to their respective objects in the instantiated object model and (via general action model ties) to the general object model.

The "has-details" inheritance relation is used to add leaf detail actions to the general action model. Figure 26 shows this relationship for concrete foundation actions. 


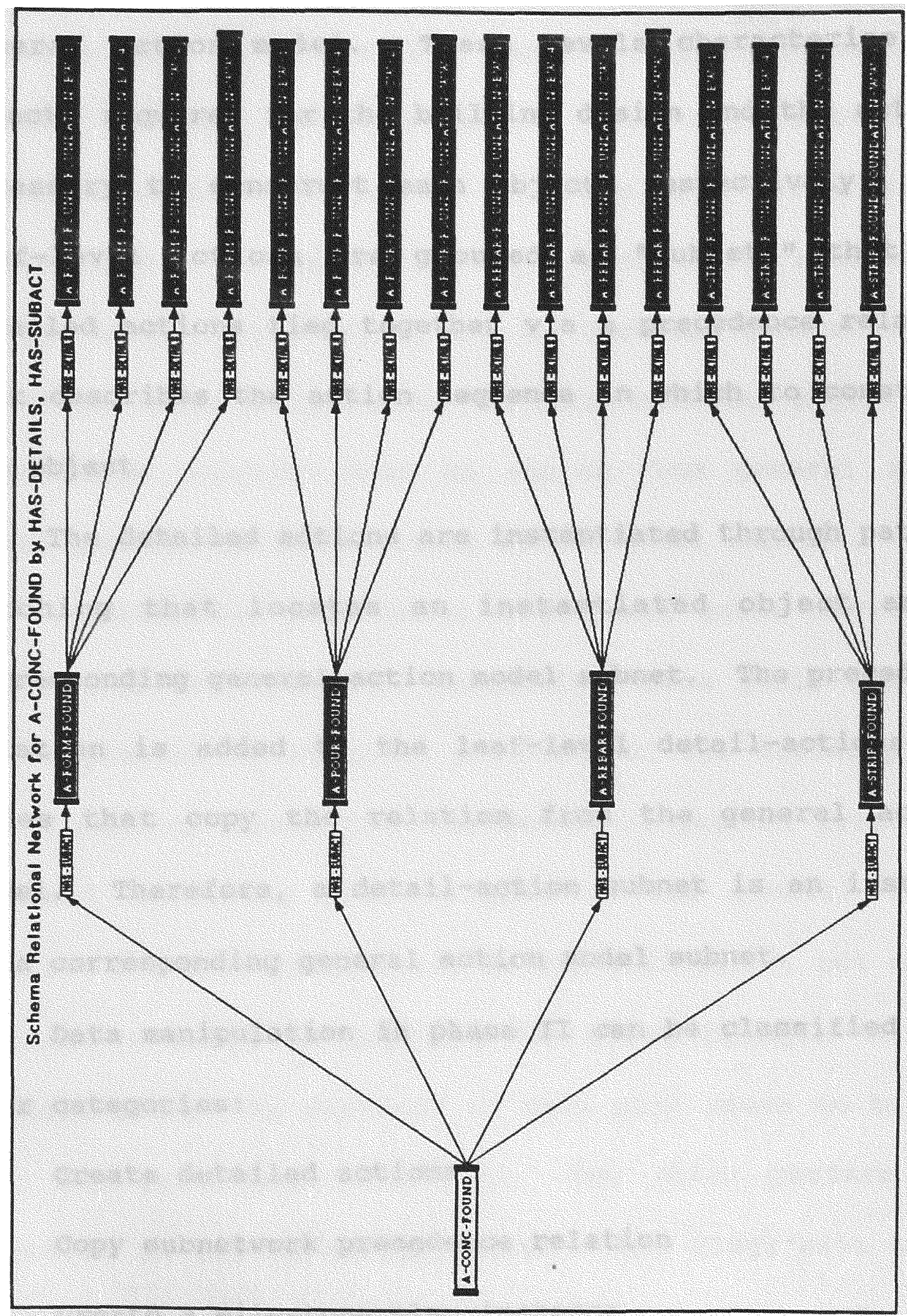

Figure 26 Relational Tie Between Action Models 
"Activities-for" associates the leaf level of the instantiated object model with the same level in the general action model. These levels characterize the objects required for the building design and the actions necessary to construct each object, respectively. The leaf-level actions are grouped as "subnets" that are detailed actions tied together via a precedence relation that describes the action sequence in which to construct one object.

The detailed actions are instantiated through pattern matching that locates an instantiated object and a corresponding general action model subnet. The precedence relation is added to the leaf-level detail-actions via rules that copy the relation from the general action model. Therefore, a detail-action subnet is an instance of a corresponding general action model subnet.

Data manipulation in phase II can be classified into four categories:
a. Create detailed actions
b. Copy subnetwork precedence relation
c. Create a milestone plan instance
d. Find head and tail nodes 
Detailed actions are created by one rule for each object in the instantiated object model. The instantiated objects have a tie to the general object model. The general action model shows how each object could be constructed. Detailed actions are created by instantiating general action model methods for each object in the instantiated object model. Once the general action model has been instantiated for each building object, the precedence relation must be copied from general action model subnets to instantiated action model subnets.

A milestone plan shows high-level goals throughout the course of a project. Generally, these goals are tied to completion of specific portions of the building that the financing institution has deemed as payment points. A general milestone plan is part of the general action model. For this project, it is not modifiable, but a user interface could be employed to allow changes to this general plan. An instance of this plan needs to be made for our particular building. Two rules perform this function in the following manner. The first rule copies each object, while creating a unique object name. The 
second rule copies the precedence relation for the general milestone plan to its instance.

The last step in phase II is to identify head and tail nodes for each subnetwork. This will be used in the next phase when inter-subnet ordering will be evaluated. Several rules perform this action by traversing each graph (through pattern matching) to find nodes with no predecessor or no successor. This will be elaborated in greater depth in the following section.

\section{Phase III Internal Specification}

At this point, instances of the general action and object models have been created. The general object model instance is a hierarchical structure whose root is the building name and leaves are the parts of the building. The action model instance is simply a new leaf layer on the general action model. All four models (two general, two instances are tied together via structural and inheritance relations.

The action model instance is a group of independent subnets that each show how to construct one object, given no outside influences. Phase III will evaluate the 
relative order of those subnets based upon building characteristics. Action order will be inferred through:
a. Building structure
b. object spatial properties
c. Material properties

A meta-planning rule specifies how to first build structural building parts from the ground up. Next, construct other, non-structural parts based upon the spatial properties of each part. In other words, if a non-structural part will be covered by another part, install the part before it is covered. There are also rules that create action ordering based upon the properties of the building materials used, i.e., water damageable materials cannot be installed until the building is dry.

Five rules perform action ordering based upon building structure. I initially attempted to minimize the number of these rules (to say one or two) by solving a more general problem. Difficulties with machine response became evident when I attempted this method. It took nearly 20 minutes to compile a more general rule, while I could load five relatively specific rules in one-third the 
time (they created smaller pattern matching networks). Each rule tests one building part type (such as foundations, walls) to see what the part is supporting. The actions for the supported part (that which is being supported) must occur later.

Since the instantiated objects have no knowledge of what they support, a protypical building was added to the general object model. This prototype contains structural information at an abstract level. Figure 27 shows this prototypical hierarchy.

(Defschema p-foundation

(supports p-found-wall))

(Defschema p-found-wall

(supports p-slab))

(Defschema p-slab

(supports p-structural-walls)

(supports p-non-structural-walls))

(Defschema p-structural-walls

(supports p-roof))

Figure 27 Building Prototype Structural

Properties

Each object in the instantiated building inherits structural properites from the general object model 
through the "is-a" inheritance relation. This knowledge is useful when applying the ordering rules described above.

One problem with adding a precedence relation through rules is that the relation is added to every possible action pair combination between subnets. If each subnet contains five actions, then 25 ties will be added between the two subnets (which is not practical). What we really want to do is to make strategic ties that minimize the number of precedences added. This can be done by isolating the head and tail nodes for each subnet and making all ties only to and from those nodes. Head and tail node identification was performed as part of phase II, but is very beneficial to this part of the project.

The spatial properties of building objects can be used in a manner similar to that applied to structural properites. The general object model contains connectivity data for each object category. Electrical cables, for instance, can be shown as part of a wall (although they could also be part of other objects as well). This connectivity information is inherited to 
object instances and can be used to infer an installation order.

Two rules were developed to show this property for electrical cables inside walls. The general rule is that if a subpart is inside a wall, then the activities for that subpart should occur after the wall structure is complete. By the same token, the subpart installation should occur before the finish activities for the wall occur. In order for this to work effectively, I classified installation activities as either rough-in or finish types. Rough-in activities include constructing the structural parts of an object. Even non-structural walls have an internal structure to support themselves. Finish activities involve installing objects that cover structural members. Plaster board is an example of a finish activity which must occur after cables have been installed in walls.

Material properties were also used to infer action order. If an object can be damaged by water, then it should not be installed until after the building is "dried-in". This state is an event in the milestone plan for the instantiated building. The milestone plan was 
created in phase II by copying a general milestone plan stored in the general action model. A rule will match the actions for all instantiated objects that have an attribute "water-damageable" (which was inherited from the general object model) and will add a precedence tie to the "building-dried-in" milestone. In this way, no water damageable object will be installed until the building is moisture free.

\section{Phase IV Internal Specification}

The graph generated during previous project phases will be analyzed based upon available resources in phase IV. This will be accomplished by assigning depth values to each node to categorize actions into groups. Beginning with the lowest numbered group, assign resources to actions until no more resources are available or no more actions remain in that group. If resources are in short supply, move actions which were neglected to the next higher group. This is an algorithmic process which was implemented using rule-based programming.

Constraint satisfaction is the purpose of this phase. The graph can be evaluated based upon resource 
availability, maximum duration and space availability. The user is prompted to input resource levels for the project and a maximum duration. Space available is calculated for each room and used to determine the maximum number of workers per room. The system will attempt to satisfy all these constraints to produce a workable schedule. If one or more constraints can not be satisfied, the user will be prompted to decide which constraint to relax so that a solution can be found.

A depth-first search was used to assign depth values to each node. This portion was not implemented using rules (although it is initiated from a rule) and is the only such algorithm in the project.

Rules, coded to perform like a "for" loop, choose the next available action to be assigned resources at the current depth. Two rules perform this action by getting actions for structural parts before those for nonstructural parts. others rules assign required resources to each action based upon resource type. For each type, a rule exists that assigns the requested amount and decrements that amount from the availability pool. 
When resources are exhausted at a given depth, remaining actions are assigned the next higher depth value, the resource availabilities are refreshed and the process repeats for the next depth. Once all depths have evaluated in this manner, an analysis of the overall project duration is made. If this constraint is not satisfied, the user is queried to relax one or more constraints before another attempt is made at finding a solution. 


\section{CHAPTER 4}

\section{TEST CASES}

Three buildings were chosen as test cases to examine whether the implemented portions of CONPLAN perform as anticipated without catastrophic failure. White box testing was performed on individual components (rules in this case) to determine their reaction to different inputs. This code driven method guarranteed that every coded statement in the final product had executed individually without failure. Black box testing was performed on the system as a whole to simulate use by an individual unfamiliar with the project's source code. White box testing in the form of logic coverage was also employed at the system level to induce errors caused by rule interaction. I attempted to create unique test cases to examine all possible rule combinations.

\section{Program Attributes to be Tested}

The purpose of testing this project is to show that the program executes without failure and to show that a plan can be developed from a two dimensional building 
representation (stored as a database). White box testing on individual rules shows their reaction to expected and unexpected keyboard input.

Backward chaining rules were used to query users for keyboard input. In most cases, "yes-or-no" questions were employed, using LISP functions that guarantee a legitimate user response. When numeric input is required, a validation function checks the input and prompts the user when unexpected characters are entered. Since it is a recursive function, it can continue its validation cycle until numeric entries are made.

The second area of testing is designed to show that the functional requirements for CONPLAN were met with the current implementation. The fundamental purpose of CONPLAN is to effectuate a construction plan from a two dimensional building image stored as a database. By examining the internal specification and source code, the algorithm I employed in this system is straight forward and will always generate a plan. It relies on characteristics of the objects gleaned from the user and stored as binary relations in a knowledge hierarchy (essentially a database with inheritance capabilities). 
This project does not purport to redefine traditional planning methods. It also performs the planning process for a very limited class of building types. One challenge to making this project a saleable product is to formulate unique methods to handle a nearly unlimited number of possible combinations of object types that could make up a building.

The major stumbling block to generating a plan using rule-based programming is that rules match every possible combination of facts that fulfill a rule's antecedent condition. To limit this effect, head and tail nodes were isolated and used to tie subnets. The two test cases chosen embody two of the three possible building configurations intended for CONPLAN. The next section will elaborate the program characteristics each test case evaluates. A third test case was employed to show the characteristics of phase 4 (resource evaluation and assignment) . 


\section{Test Cases Chosen}

Test case 1 is a building constructed of concrete block and concrete structural walls, wood-frame nonstructural walls, a gable truss roof, concrete foundations and a concrete slab. This example will test user interface rules associated with each of these categories as well as plan generation rules in phases 2 and 3. These include rules for generated structural walls, nonstructural walls, those for a gable roof, as well as foundation and slab rules.

The building for test case 2 is constructed of concrete block structural walls, wood-frame nonstructural walls, a gable and hip roof, concrete foundations and a concrete slab. This example tests hip roof structure rules which were not tested in the previous example.

Phase 4 could not be tested in a manner consistent with the two previous test cases. Database characteristics required for phase 4 were not yet implemented in the previous phases. As such, a unique building had to be created that contained all the requirements. Phases 1 to 3 were used to generate a sample building (called "DM") and the schemata were saved 
to a file. The file was then manually modified to reflect the required database changes that were necessary to test phase 4. 


\section{CHAPTER 5}

\section{SUMMARY}

PROJCON is designed to maximize management decisionmaking capabilities for planning, scheduling, chronicling and analysis. Strategic plans can be specified through a user interface and tactical implementation of those strategic plans can be changed to reflect current job conditions. Plan generation through graphical object specification is a new approach and allows designers to immediately assess impact of design decisions on construction of the project. The output of the planning subsystem is a partially ordered graph. During the scheduling phase, the partially ordered graph is evaluated based upon resource, workspace and strategic planning constraints. Interactive graphics, used for schedule chronicling and analysis, allow efficient schedule modification.

CONPLAN is the planning portion of PROJCON that utilizes a graphical user interface to generate a hierarchically represented database of facts that describe a building from a strictly defined class. It then creates 
a plan by instantiating predefined subplans for each object defined in the database and then evaluates the order of the subplans based upon interobject relationships. Subplan order can be infered through structural, spatial and material properties stored in the database.

structural properties are inherited to instantiated objects. Also, the objects "supported" by structural objects can be found through a protypical building structure stored in the general object model. For example, if an instance of a wall is a structural wall, then that wall instance supports the roof. spatial properties can help infer subplan order by applying rules that check for objects that "cover" other objects, thereby suggesting their installation order. Material properties, such as water damageable materials, must be installed after the building is "dried-in" (a step in the milestone plan).

The implementation of CONPLAN taught me a tremendous amount about problem complexity and the difficulty of modeling that complexity in a limited amount of disk space. The symbolics Lisp Machine employed for this 
project had a limited amount of disk space, which limits the amount of virtual memory that is available. This limitation forced the project to be broken into smaller pieces in order to run. Each phase is loaded independently of each other, run and removed from memory before the next phase is loaded.

Even if a more reasonable disk size was available, the reality is that it is not feasible to create a general model for a complicated domain (such as the domain used for this project). A solution is to use conventional hardware and software where applicable and expert systems for small specialized evaluation tools. Future work could explore designs for PROJCON that include conventional as well as expert technologies. Computer aided design (CAD) database models coupled with rule-based programming could be one alternative. 


\section{REFERENCES :}

1. Art Programing Reference Manual, Inference Corporation, Los Angeles, California, 1986.

2. Baker, $K$. R. Introduction to sequencing and Scheduling, New York, NY, John Wiley, 1974.

3. Barrie, D. S. and Paulson, B. C. Professional Construction Management, McGraw-Hill, Inc., 1984.

4. Brachman, R. J. On the Epistemological status of Semantic Networks. In NV Findler, Ed., Associative Networks: Representation and Use of Knowledge by Computers, Academic Press, New York, NY, 1979: 3-50.

5. Carbonell, J. R. Mixed-Initiative Man-computer Instructional Dialogs, BBN Report no. 1971. Bolt, Beranek and Newman, Cambridge, MA, 1970.

6. Cohen, P.R., Feigenbaum, E.A. The Handbook of Artificial Intelligence, Volume 3, HeurisTech Press, 1982.

7. Conway, R., Maxwell, W. L., Mcclain, J. O., Worone, S. L., User's Guide to XCELLt Factory Modeling system, The scientific Press, 1987.

8. Corkill, D. D. Hierarchical Planning in a Distributed Environment, Proceedings of the sixth International Conference on Artificial Intelligence, 1979: $168-175$.

9. Dekleer, J. An Assumption-Based TMS, Artificial Intelligence, Vol.28, No 1, 1986.

10. Fox, M. S. Constraint Directed Search: A Case Study of Job-Shop Scheduling. Ph-D Th., Computer Science Dept, Carnegie Melion University, Pittsburgh, PA $15213,1983$. 
11. Fox, M. S. Observations on the Role of constraints in Problem solving, Proceedings of the Annual Conference of the canadian society for computational Studies of Intelligence, May 1986, Montreal, Quebec.

12. Gary, M. R. and Johnson, D. S. Computers and Intractability. A Guide to the Theory of NPCompleteness, W. H. Freeman and Company, New York, NY, 1979.

13. Gray, C., Little, J., Expert system development for predicting time and cost of construction during initial design, First International Expert systems Conference, London, 1986.

14. Genesereth, M. and Nilsson, N. J. Logical Foundations of Artificial Intelligence, Morgan Kaufmann Publishing Company, 1987.

15. Hayes-Roth, B. A. Blackboard Architecture for Control, Artificial Intelligence Journal, Vol 26, 1985: 251-321.

16. Hendrickson, C., Martinelli, D., Rehak, D. Hierarchical Rule-Based Activity Duration Estimation, Working paper, Dept. of Civil Engineering, CarnegieMellon University, 1986.

17. Hendrix, G. G. Expanding the Utility of semantic Networks through Partitioning, Fourth International Joint Conference on Artificial Intelligence, Tiblisi, USSR, IJCAI, 1975.

18. Kim, S. S., Maher, M. L., Levitt, R. E., Rooney, M. F., Siller, T. J., Richie, S. G., Survey of the state-of-the-Art Expert/knowledge Based systems in Civil Engineering, USA-CERL special report $\mathrm{P}-87 / 01$, October, 1986.

19. Levitt, R. E. and Kunz, J. C. Using Knowledge of Construction and Project Management for Automated Schedule Updating, Project Management Journal, Vol 16 , No 5, December 1985. 
20. Levitt, R. E. and Kunz, J. C. Using Artificial Intelligence Techniques to support project Management, The Journal of Artificial Intelligence in Engineering, Design, Analysis and Manufacturing, 1987.

21. Leustra, J. K., Sequencing by Enumerative Methods, Mathematisch Centrum, Amsterdam, Ph.D. Dissertation, 1977.

22. Levy, F. K., Rosenbaum, J. H., west, J. D., The $A B C$ of the Critical Path Method, Harvard Business Review, oct. 1963 .

23. Malcolm, D. G., Rosenbaum, G. L., Clark, C. E. Application of Technique for Research Development Program Evaluation, Operation Research, Sept., 1959.

24. Minsky, M. A Framework for Representing Knowledge, in The Psychology of Computer Vision. P. Winston (Ed.), New York: McGraw-Hill, 1975.

25. National Electric Code, National Fire Protection Association, Battery Park, Quincy, Massachussetts, 1980 .

26. Nii, H. P. Blackboard Systems: The Blackboard Model of Problem Solving and the Evolution of Blackboard Architectures, The AI Magazine, Summer 1986: 38-106.

27. Nilsson, N. J. Principles of Artificial Intelligence, Tioga Publishing Co., 1980.

28. O'Connor, M. J., Delagarza, J. M. and Ibbs, C. W. An Expert System for Construction Schedule Analysis, in Expert Systems in Civil Engineering, edited by Kostem and Maher, ASCE, April 1986.

29. Quillian, M. R. Semantic Memory. Ph.D. Thesis, Carnegie Mellon University, Pittsburgh, Pa, 1966.

30. Rinnooy-Kan, A. Machine Scheduling Problem Classification, Complexity and Configuration, Ph.D. Thesis, Leyden, Steufert Kroese, 1976. 
31. Sacerdoti, E. D. Planning in a Hierarchy of Abstract Spaces, Third International Conference on Artificial Intelligence, IJCAI, 1973: 412-422.

32. Sathi, A., Fox, M. S. and Greenberg, M. Representation of Activity Knowledge for project Management, Robotics Institute, Carnegie Mellon University, 1985.

33. Sathi, A., Morton, T. E., Roth, S. F. Callisto: An Inteligent Project Management system, The AI Magazine, Winter 1986: 34-52.

34. Schubert, L. K. Extending the Expressive Power of Semantic Networks, Artificial Intelligence 7, 1976: 163-198.

35. Shapiro, S. C. The MIND System: A Data Structure for Semantic Information Processing, Tech report $R-$ 837-PR, Rand Corporation, 1971.

36. Simon, H. A. The structure of Ill structured Problems, Artificial Intelligence 4, North-Holland Publishing, 1973: 181-201.

37. South Florida Building Code, Board of county Commissioners, Dade County, Florida, 1984.

38. Smith, S. F. Exploiting Temporal Knowledge to organize Constraints. Tech. Rept. CMU-RI-TR-83-12, ISL, Robotics Institute, Carnegie Mellon University, Pittsburgh, PA 15213, 1983.

39. Steffan, M. S. A Survey of Artificial IntelligenceBased Scheduling systems. Proceedings of Fall Industrial Engineering Conference, Boston, MA, 1986.

40. Stefik, M. An Examination of a Frame-structured Representation System. Proceedings of the Sixth Joint Conference on Artificial Intelligence, Tokyo, Japan, IJCAI, 1979. 
41. Tate, A. Generating Project Networks. The Fifth International Joint conference on Artificial Intelligence, IJCAI, 1977: 888-893.

42. Waterman, D. A. and Hayes-Roth, F. Pattern Directed Inference Systems, Academic Press, 1978.

43. Wilkins, D. E. Domain-independent Planning: Representation and Plan Generation, Artificial Intelligence 22, Elsevier Science Publishers, 1984: 269-301. 
VITA

Title of Thesis

An Expert Project Control system for construction Management

Full Name: Kermit H. Gates III

Place and Date of Birth

Wurzburg, Germany. October 4, 1959.

Elementary and Secondary Education

Elementary Education: various schools associated with the armed forces.

Secondary Education: Walt Whitman HS, Bethesda, Maryland.

Colleges and Universities - Years Attended and Degrees

University of Florida, school of Building construction, 1977-82, Bachelor in Building construction.

Florida International University, school of Computer Science, 1984-88, Master of science in Computer science.

Membership in Learned or Honorary Societies

Sigma Lambda Chi, Construction Honor Society.

Publications

Gates, K. H. and Lenart, M. "An Expert system for project controls in construction Mangement", Proceedings of the Third International conference on Applications of Artificial Intelligence in Engineering, Stanford, California, 1988. 
Gates, K. H. and Lenart, M. "Projcon: Construction Management Expert system", Applied Artificial Intelligence Reporter, December 1 , 1987 .

Major Department

School of computer science 